\title{
Suspension of flexible cylinders in laminar liquid flow
}

\author{
J.J. Derksen
}

School of Engineering, University of Aberdeen, Aberdeen, UK

jderksen@abdn.ac.uk

Submitted to AIChE Journal - August 2019

Resubmission - December 2019

Revision - February 2020

Accepted - February 2020

\begin{abstract}
A methodology for particle-resolved simulation of dense suspensions of flexible cylindrical particles in Newtonian liquid flow is described. It is based on the lattice-Boltzmann method for solving the liquid flow and an immersed boundary method for imposing no-slip at the particle surfaces and providing the distribution of liquid-solid interaction forces over the particle surfaces. These forces - along with contact forces - translate, rotate as well as bend the cylindrical particles. Verification tests have been performed for a single cylinder settling and deforming under gravity at a low Reynolds number. The method has been applied to a clamped flexible cylinder in microchannel flow for which experimental data are available. It then is used to investigate the behavior of hundreds of flexible cylinders with length over diameter aspect ratios of 4 and 6 in a container agitated by an impeller at a Reynolds number of 87 which implies laminar flow. The overall solids volume fraction is $15 \%$. We study the effect of the bending stiffness of the particles on the solids suspension process, on the extent of particle deformation as well as on the torque required to spin the impeller.
\end{abstract}

\section{Keywords}

Liquid-solids flow; particle-resolved simulations; flexible particles; lattice-Boltzmann method; bending stiffness. 


\section{Introduction}

Fibrous materials are commonplace in a myriad of applications. In the very common form of paper, but also - for example - as reinforced composite materials [1] and for energy storage as "fiber supercapacitors" [2]. Producing such materials often involves stages where solids - in the form of fibers - are suspended in liquid and are transported as a dense two-phase flow. Flow of dense fiber suspensions is also encountered in biomass conversion where materials of a fibrous nature (e.g. wood, waste from food crops) are processed for energy and materials production [3,4]. Therefore, predicting the flow dynamics of fiber suspensions, specifically in complex configurations as often seen in process equipment, has practical relevance. The approach to fiber suspension flow we have taken in recent publications $[5,6]$ is based on particle-resolved simulation. Particles of cylindrical shape are placed in a domain containing a Newtonian liquid. The flow of liquid is simulated with a resolution much finer than the size of the cylinders so that the no-slip condition can be explicitly imposed at their surface and the hydrodynamic forces and torques on the cylinders directly calculated. These then induce translation and rotation of the cylinders and this motion is fed back to the liquid as - updated - no-slip conditions. This way an intimate dynamic coupling between liquid flow and particle motion is established that directly takes into account the (cylindrical) shape of the particles as well as the microstructure they are organized in.

The previous simulations assumed the cylinders to be rigid $[5,6]$. In the present paper an approach is outlined to include deformation of the cylindrical particles in the numerical procedure. The reason for doing this is driven by applications -in many cases fibers in materials processing are flexible - as well as by curiosity. Regarding the latter, we want to explore under what circumstances flexibility of particles has an impact on the overall flow behavior, for instance in terms of directly measurable, macroscopic flow quantities.

As we will see, our existing computational framework for particle-resolved simulations of dense suspensions involving non-spherical rigid particles [5,6], is well suited for an extension towards particle deformation. Given that in our methodology no-slip conditions at particle surfaces are imposed through a forcing variant of the immersed boundary method (IBM) [7], the distribution of forces over the solid 
surfaces is directly available in the simulation. These forces, along with forces arising from contact between particles and between particles and internals in the flow domain (baffles, impellers), are not only responsible for particle motion (translation and rotation) but also for particle deformation. This paper only considers a bending deformation. This is for simplicity but also since bending is the most significant deformation of slender cylindrical particles.

When it comes to direct simulations of solid-fluid flow with rigid particles of non-spherical shape, recent work has made major strides in the accurate representation of the way particles dynamically interact with fluid $[8,9]$. There is limited literature on the subject of dense suspensions of deformable, non-spherical particles in fluid flow, the exception being detailed simulations of blood flow: Direct simulations of red blood cells (RBC's) suspended in plasma that include the deformability of its membranes have - for instance - been reported by $\mathrm{Wu} \&$ Aidun [10]. There, deformation of the RBC membrane is dealt with through finite element analysis, while fluid flow is solved by the lattice-Boltzmann method.

Experimental and computational progress on flexible fibers in fluid flow has recently been reviewed by du Roure et al [11] with an emphasis on microscopic systems. In one type of simulation approach fibers are represented by chains of connected spheres $[12,13,14]$. This allows for very strong deformations of the fibers. It does, at the same time, not very accurately represent the actual shape of the fibers and - therefore the way it interacts with the fluid. The immersed boundary method - as also used in this paper - has been effectively implemented in parallel computer code by Wiens and Stockie [15] and applied to flow systems involving single and multiple slender flexible fibers.

The aim of this paper is to introduce and explain in detail an extension of previous work on particleresolved simulations of cylinder suspensions [5,6] towards particles with finite bending stiffness with the deformed particle shape fully accounted for by the IBM. We also aim for demonstrating the potential of the method for applications with complex flow conditions by showing results for dense suspension of hundreds of flexible cylinders in a non-trivial - albeit laminar - liquid flow generated by a revolving impeller. It has been investigated how bending stiffness impacts the overall flow behavior of this system. 
It is important to experimentally validate the proposed numerical procedure, preferably at the level of individual particles and the way they deform under well-defined flow conditions. Such detailed experimental work is relatively scarce [11]. In the current paper experimental work by Wexler et al [16] on a single fiber bending in a micro channel flow has been used for this purpose. We also have performed a number of verifications - including checking grid effects as well as assessing what resolution is required for the deformation calculations - for a single settling cylinder at low Reynolds number. Given the need for detailed validation, a secondary aim of this paper is to engage experimentalists to work on highly resolved visualizations of flexible fibers in fluid flow.

\section{Flow systems}

We perform simulations in three-dimensional rectangular flow domains. They are bounded by solid, no-slip walls all around, except for one system that consists of a micro channel (Hele-Shaw cell) that has been simulated with periodic boundaries in the streamwise direction. The domains are filled with a Newtonian liquid with density $\rho$ and kinematic viscosity $\nu$. In addition, the domains contain one or more solid cylindrical particles with diameter $d$, length $\ell$, and density $\rho_{p}>\rho$ (particle volume is denoted as $V_{p}=\frac{1}{4} \pi d^{2} \ell$ and mass as $\left.m=\rho_{p} V_{p}\right)$. The cylinders are bendable, i.e. they have a bending stiffness $E I_{c s}$ with $E$ Young's modulus and $I_{c s}$ the moment of inertia of the cross sectional area of the cylinder ( $I_{c s}=\pi d^{4} / 64$ ). Bending is the only allowed deformation; the cylinders cannot be stretched, compressed or twisted.

Three flow configurations have been considered in this paper: (1) Single horizontally oriented flexible cylinders settling in a tall closed box have been studied for the purpose of verifying the simulation procedure. (2) A Hele-Shaw cell with one cylinder with one of its ends attached to a side wall - a similar system has been considered in experiments [16] - that we use for validation. (3) Multiple flexible cylinders immersed in the liquid flow generated by a revolving impeller have been studied as an example of a manyparticle system in complex flow. The configurations - including nomenclature of dimensions and coordinate 
systems - are defined in Figure 1. In systems (1) and (3) the effect of gravity is important; there gravity acts in the negative $z$-direction: $\mathbf{g}=-g \mathbf{e}_{\mathbf{z}}$. The mixing tank is agitated by a pitched-blade turbine (PBT) that pumps in the downward (negative $z$ ) direction. The impeller has a diameter $D$ and makes $N$ revolutions per unit time.

All three systems are being characterized in dimensionless terms. Where gravity is important, the particles-fluid combination is characterized by the Archimedes number $\operatorname{Ar}=(\gamma-1) g d^{3} / \nu^{2}$ with $\gamma=\rho_{p} / \rho$ the density ratio. The bending stiffness has been non-dimensionalized as $\sigma=\frac{E I_{c s}}{\rho(\gamma-1) g d^{5}}$. In the HeleShaw cell, that is operated at low Reynolds numbers, viscous drag is combined with bending stiffness in the dimensionless parameter $\chi=\frac{\rho \nu U \ell^{2} d}{E I_{c s}}$ with $U$ the superficial velocity in the cell. The Reynolds number associated with the impeller agitating the liquid is defined as $\operatorname{Re}=N D^{2} / \nu$. A Shields number reflects the competition between agitation and net gravity acting on the particles: $\theta=N^{2} D^{2} /(g(\gamma-1) d)$. The overall solids volume fraction in the mixing tank is $\langle\phi\rangle=\frac{n V_{p}}{H T^{2}}$, with $n$ the number of particles and $H T^{2}$ the container volume (see Figure 1). The system's geometry is characterized by a number of size ratios; the most notable aspect ratio is $\ell / d$, the cylinder's length over its diameter.

\section{Numerical methods}

This paper extends the simulations on dense liquid-solids suspensions with rigid particles of cylindrical shape as discussed in $[5,6]$ towards cylinders with a finite bending stiffness. We begin this section with a brief overview of the numerical approach (details in [5]) and then zoom in on how bending has been implemented as well as discuss the assumptions and limitations of the implementation.

\section{Overview of the simulation procedure}

The lattice-Boltzmann (LB) method $[17,18]$ has been used as the fluid flow solver. It operates on a uniform, cubic grid with spacing $\Delta$. The flow variables (fluid velocity $\mathbf{u}$ and pressure $p$ ) are derived from discrete 
velocity distribution functions that are the primitive variables of the method and are updated according to the LB equation that involves a collision and a streaming step [19]. The system discretely evolves in time with a time step $\Delta t$. Flow variables are defined in the center of each cubic grid cell. The specific LB scheme used in this work is due to $[20,21]$.

In order to represent solid (that is, no-slip) surfaces inside the flow domain (particle surfaces as well as the impeller) an immersed boundary method (IBM) has been developed previously [22]. Solid surfaces are defined by collections of closely spaced off-grid points (nearest neighbor spacing of these points $\approx 0.5 \Delta$ ). At these so-called marker points we determine forces on the fluid such that the local fluid velocity (linearly interpolated from the lattice) closely approximates the local surface velocity so as to achieve no-slip. The IBM thus provides us with the distribution of fluid-solid interaction forces over the solid surfaces. Integrating the forces over the surface of each particle gives the total hydrodynamic force and torque on that particle that - along with other forces such as gravity and contact forces - are used to integrate the linear and rotational equations of motion of each particle. Now that particles are deformable, the distribution of forces over the particles will also be used to determine their bending deformation. This will be explained in more detail in the next sub-section.

In addition to the particles, also the impeller has been represented through the IBM. The bounding walls of the flow domain are aligned with the grid. At these walls the halfway bounce-back rule has been applied to the velocity distribution functions [19] in order to achieve no-slip.

Given our interest in dense suspensions, it is expected that collisions (between particles, between particles and the impeller, and between particles and the bounding walls) are frequent. In [5] we have detailed our approach for particle-particle collisions. Collision detection is based on the IBM marker points: two marker points lying on two different particle surfaces coming within a certain distance triggers a repulsive elastic (spring) force between the particles that prevent them from overlapping. In addition, a damping force proportional to the velocity difference of the two marker points gets activated to mimic lubrication effects between the two closely spaced solid surfaces. In the context of the discrete element method (DEM) this approach for collision detection is known as discrete function representation [23]. As 
shown in [6], the method as developed for particle-particle collisions can be straightforwardly extended towards collisions between a particle and the impeller as well as between a particle and a bounding wall.

To update the orientation of particles, quaternions have been used [24]: each particle is equipped with a unit quaternion $q=\left(q_{0}, \mathbf{q}\right)$ that fully defines the way it is oriented in three-dimensional space. After updating the angular velocity $\omega_{p}$ of each particle based on the Euler equations for the dynamics of particle rotation [25], the quaternions are updated according to an exact solution [26]: $q^{(k+1)}=\left(\cos \left(\frac{1}{2} \Delta t\right), \boldsymbol{\omega}_{\mathbf{p}} \sin \left(\frac{1}{2} \Delta t\right)\right) \circ q^{(k)}$ where the index $(k)$ denotes the time step number, and the symbol $\circ$ the quaternion multiplication [24]. The same time step as used in the LB method has been used for the solid particle updates.

Quaternions enable solving the dynamics of the cylindrical particles in a reference frame $\left(x_{1}, x_{2}, x_{3}\right)$ attached to the particle, see Figure 2. In this reference frame, the moment of inertia tensor of the (rigid) particle is constant and diagonal with diagonal components $I_{11}=\frac{1}{8} m d^{2}$ and $I_{22}=I_{33}=m\left(\frac{1}{16} d^{2}+\frac{1}{12} \ell^{2}\right)$. Transferring information between the inertial reference frame $(x, y, z)$ and a particle reference frame is facilitated by the quaternion associated to the particle: $\mathbf{y}=\bar{q} \circ(0, \mathbf{x}) \circ q$ with the vector $\mathbf{x}$ defined in the inertial frame and $\mathbf{y}$ defined in the particle frame, while $\bar{q}=\left(q_{0},-\mathbf{q}\right)$ [27].

\section{Cylinders with finite bending stiffness}

The simulation procedure as sketched above has been used for suspensions with rigid cylindrical particles $[5,6]$. The IBM as well as the collision algorithm provide us with the distribution of forces over the surfaces of the particles at any moment in time. With this information the deformation of non-rigid particles can be determined. This is explored in this paper for cylindrical particles with a finite bending stiffness.

In the terminology of structural mechanics [28], the cylinder is a beam with a bending stiffness $E I_{c s}$ that is deflected by a distributed load (force per unit length along the beam) $a_{2}\left(x_{1}\right)$ and $a_{3}\left(x_{1}\right)$ in the two lateral directions $x_{2}$ and $x_{3}$ respectively (see Figure 2), with $x_{1}$ the coordinate along the centerline of the 
beam. The load in the $x_{1}$ direction is irrelevant for bending; it would be relevant for stretching or compression which are, however, deformations not considered in this study.

The force distribution over the cylinder surface that is the result of the IBM and the particle contact algorithm has three consequences: (1) it accelerates the particle (in a linear and angular sense); (2) it opposes net gravity; (3) it bends the particle. In the $x_{2}$ and $x_{3}$ direction this implies

$$
\begin{aligned}
& b_{2}\left(x_{1}\right)=a_{2}\left(x_{1}\right)+\alpha\left(\frac{d u_{p 2}}{d t}+x_{1} \frac{d \omega_{p 3}}{d t}-g_{2}(\gamma-1)\right) \\
& b_{3}\left(x_{1}\right)=a_{3}\left(x_{1}\right)+\alpha\left(\frac{d u_{p 3}}{d t}-x_{1} \frac{d \omega_{p 2}}{d t}-g_{3}(\gamma-1)\right)
\end{aligned}
$$

with $b_{2}\left(x_{1}\right)$ and $b_{3}\left(x_{1}\right)$ the total force per unit length at axial location $x_{1}$ in the $x_{2}$ and $x_{3}$ direction respectively, $\alpha=m / \ell$ the mass per unit length of the cylinder, $u_{p 2}$ and $u_{p 3}$ components of the linear velocity of the particle, $\omega_{p 2}$ and $\omega_{p 3}$ angular velocity components, and $g_{2}$ and $g_{3}$ gravitational acceleration in the $x_{2}$ and $x_{3}$ direction respectively. Given that the particle acceleration (linear and angular) is solved separately in the simulation procedure, Eqs. $1 \& 2$ allow - at every moment in time for each particle - the determination of the load distributions $a_{2}$ and $a_{3}$ that bend the particle.

In a quasi-static approximation, the load distributions relate to bending moments $M_{2}$ and $M_{3}$ according to [28]

$$
a_{2}=\frac{d^{2} M_{2}}{d x_{1}^{2}} \quad a_{3}=\frac{d^{2} M_{3}}{d x_{1}^{2}}
$$

For freely moving particles both ends of the cylinder are unconstrained. Then the boundary conditions for these two second-order ordinary differential equations (ODE's) are that at $x_{1}=-\frac{1}{2} \ell$ and $x_{1}=\frac{1}{2} \ell$ the bending moments are zero: $M_{2}\left(x_{1}= \pm \frac{1}{2} \ell\right)=M_{3}\left(x_{1}= \pm \frac{1}{2} \ell\right)=0$.

The deflections of the beam $\left(w_{2}\right.$ and $\left.w_{3}\right)$ obey [28]

$$
M_{2}=E I_{c s} \frac{d^{2} w_{2}}{d x_{1}^{2}} \quad M_{3}=E I_{c s} \frac{d^{2} w_{3}}{d x_{1}^{2}}
$$


in the limit of small deflections, i.e. if $\left|w_{2}\right| \ll d$ and $\left|w_{3}\right| \ll d$. Solving for $w_{2}$ and $w_{3}$ requires again two boundary conditions each. Since the overall translation and rotation of the cylinder are updated by solving the dynamic equations of the cylinder in its entirety, the deflections are not allowed to add additional overall translation or rotation. Therefore the average deflection as well as the average deflection gradient must be zero. For $w_{2}: \int_{-\ell / 2}^{\ell / 2} w_{2} d x_{1}=0$ and $\int_{-\ell / 2}^{\ell / 2} \frac{d w_{2}}{d x_{1}} d x_{1}=0$. The latter implies $w_{2}(-\ell / 2)=w_{2}(\ell / 2)$. The same boundary conditions apply to $w_{3}$.

The sets of ODE's (Eqs. $3 \& 4$ ) are solved through finite differences. The cylinder is divided in $n_{s}$ equally sized segments with length $\Delta x_{1}=\ell / n_{s}$. The second derivatives are discretized according to a central scheme. As an example, for $M_{2}$ this reads $\left.\frac{d^{2} M_{2}}{d x_{1}^{2}}\right|_{i}=\frac{M_{2, i+1}+M_{2, i-1}-2 M_{2, i}}{\Delta x_{1}^{2}}+O\left(\Delta x_{1}^{2}\right)$ with $i=1 \ldots n_{s}$ and the $i$-nodes located in the middle of each segment (see Figure 3a). For each of the ODE's this leads to a linear system of equations of size $n_{s}$ in the nodal values of $M_{2}, M_{3}, w_{2}, w_{3}$ that is solved directly. The bending loads $a_{2}$ and $a_{3}$ are determined by first integrating the hydrodynamic and contact forces over each segment of the cylinder so as to calculate $b_{2}$ and $b_{3}$ and then apply Eqs. $1 \& 2$.

Once we have calculated the deflections $w_{2}$ and $w_{3}$ for a cylinder, its shape in terms of marker points needs to be adjusted in order to apply the IBM as well as collision detection and execution at the surfaces of the deformed cylinders. The marker points are grouped per segment (see Figure $3 \mathrm{~b}$ ) and the deflection is dealt with as a translation and rotation per segment. Figure 3 illustrates how this has been implemented where - for clarity of illustration - in Figure 3a we only consider a deflection $w_{2}$ in the $x_{2}$ direction.

Numerically solving Eq. 4 results in the nodal deflection values $w_{2, i}$. These are first translated into edge deflection values $w_{2, i}^{(e)}$ (defined in Figure 3a) through linear interpolation as well as using the boundary conditions. Starting from the middle of the cylinder (at $x_{1}=0$ ), the edge locations on the centerline are displaced by $w_{2, i}^{(e)}$ in the $x_{2}$ direction and the segment centerline is rotated by an angle asin $\left(\frac{w_{2, i}^{(e)}-w_{2, i-1}^{(e)}}{\Delta x_{1}}\right)$. In 
order to keep the segments on the deformed centerline (the red line pieces in Figure 3a) connected with neighboring segments, the segments are also shifted in the $x_{1}$ direction. The same shift and rotation as applied to the centerline segments is then also applied to the marker points of each segment (where the rotations again make use of quaternions). Figure 3c shows an example of how a deformed cylinder is represented by marker points. Since the IBM as well as collision handling are using the marker point on the deformed cylinder, the fluid-solid and solid-solid interactions are directly taking into account the evolving shape of the cylindrical particles.

There are a number of assumptions and approximations that we would like to highlight here; most of them have been adopted for simplicity and computational efficiency and therefore there is clearly room for future refinements of the methodology. (1) Only bending as a deformation is allowed. (2) Equation 3 is based on a quasi-static assumption. This implies that we assume that fluctuations with which the deforming load $\left(a_{2}, a_{3}\right)$ changes in time are slower than the eigen-fluctuations of the flexible beam. The load fluctuations relate to flow time scales. An order of magnitude estimate of the beam's bending eigenfrequencies is $f_{\text {bend }}=\sqrt{\frac{E I}{\ell^{3} m}}$ [29]. We will be comparing time scales when discussing the simulation results. (3) Equation 4 is valid for small deflections $\left(\left|w_{2}\right| \ll d\right.$ and $\left.\left|w_{3}\right| \ll d\right)$. As we will see from the simulation results, this condition is challenging for the low end of the range of the flexibility parameter $\sigma=\frac{E I_{c s}}{\rho(\gamma-1) g d^{5}}$ and for the high end of $\chi=\frac{\rho \nu U \ell^{2} d}{E I_{c s}}$ as investigated in this paper. Two additional approximations originate from the small-deflections assumption: (3a) In updating the equations of rotational motion of the cylinders, the moment of inertia tensor of the non-deformed cylinder has been used. (3b) The velocity directly associated to deflections, i.e. the change in deflection per unit time $\left(d w_{2} / d t\right.$ and $\left.d w_{3} / d t\right)$, is not considered when imposing no-slip by means of the IBM.

\section{Set-up of the simulations}


Throughout this paper the same liquid, cylinder diameter and cylinder density have been used. Since also gravitational acceleration has not been varied, the Archimedes number is constant. It has value $\mathrm{Ar}=4.15$. The density ratio is $\gamma=1.25$. Most simulations deal with cylinders with $\ell / d=4$, except for one agitated tank simulation that has $\ell / d=6$ as well as the cylinder in the Hele-Shaw cell that has an aspect ratio in line with the available experimental data. The simulations focus on the impact the bending stiffness parameters $\sigma$ and $\chi$ have on the behavior of the solid-liquid flow system. Variation of $\sigma$ is in the range $0.5 \leq \sigma \leq 50$ in the settling simulations, and $5 \leq \sigma \leq 150$ in the mixing tank simulations; furthermore $10^{-4}<\chi<10^{-1}$. The flow of liquid in the mixing tank is laminar with a fixed value of the impeller-based Reynolds number: $\mathrm{Re}=87$. The mixing tank simulations all have a Shields number $\theta=9.08$. The same flow geometry and conditions have been applied in a previous paper [6] that studied rigid cylinders in a mixing tank. An overview of the dimensionless numbers used in this paper is given in Table 1.

As the default spatial resolution of the simulations, the diameter of a cylinder spans 12 lattice spacings: $d=12 \Delta$. Grid sensitivity has been assessed previously [5] and also in the settling and Hele-Shaw cell simulations in the current paper. Here by comparing results with the default resolution to those with $d=16 \Delta$ and $d=24 \Delta$. The default number of segments when solving the bending-related ODE's (Eqs. $3 \&$ 4) for $\ell / d=4$ is $n_{s}=20$. Also the sensitivity of this choice has been investigated.

The $T \times T \times H$ agitated tank has been discretized with $n x \times n y \times n z$ cubic cells with $n x=n y=251$ and $n z=240$. In this domain, the impeller is represented by a collection of marker points and associated outward unit normal vectors at its surface. The marker points revolve around the $z$-axis such that the impeller makes one revolution in 4000 time steps $(N=1 /(4000 \Delta t))$.

Collision detection and execution of collisions (particle-particle, particle-impeller as well as particlewall) is identical as in [6], including the values of the coefficients used in the elastic force and damping force expressions. For completeness, Table 2 summarizes the elastic and damping force expressions, the parameters as used in the expressions and the definition of the relative location of the two marker points 
between which the forces get activated. These are located on two different particles in case of a particleparticle collision; one of them is on the impeller in case of an impeller-particle collision.

For initialization of the simulations we first randomly distribute particles in a non-overlapping way in the $T \times T \times H$ flow domain, without the impeller being present. The number of particles has been chosen so as to achieve a solids volume fraction of $\langle\phi\rangle=0.148$; if $\ell / d=4$ there are $n=411$ particles in the tank, for $\ell / d=6, n=274$. The particles settle so that they eventually form a granular bed on the bottom. Then the impeller is installed and is set to rotate. In the first 4000 time steps the impeller speed ramps up linearly, after that it has its steady value of $N=1 /(4000 \Delta t)$.

\section{Results}

\section{Single cylinder settling - verifications}

A single cylinder with $\ell / d=4$ is placed horizontally in a rectangular box filled with liquid and closed off all around. At time zero - when cylinder and liquid have zero velocity - gravity is switched on and the particle settles and while settling bends, see Figure 4 that shows snapshots of particle location and shape as well as the liquid velocity magnitude contours in a cross sectional plane. Quickly (within one viscous time $\left.d^{2} / \nu\right)$ the particle bends to a steady shape. In the middle panel of Figure 4, the segments constituting the bended cylinder have been made visible. Upon approaching the bottom the cylinder rectifies itself while liquid is being squeezed out of the space between bottom and particle. This evolution is also shown in Figure 5 in terms of time series of the Reynolds number based on the instantaneous settling velocity $\operatorname{Re}_{s}=u_{s} d / \nu$ and the deflection relative to the cylinder diameter $\Delta w / d$ with $\Delta w$ the difference between the deflection at the end points and in the middle of the cylinder $(\Delta w=w(0)-w(\ell / 2)$ where it is reminded that $w(-\ell / 2)=w(\ell / 2)$ is a boundary condition). We observe a weak coupling between settling speed and deformation: the most flexible cylinder settles fastest. The change of shape affects the drag coefficient and therefore the settling velocity. The time it takes for the particle to deform is shorter than the time for it to reach a steady velocity. As expected, the deformation strongly depends on the stiffness of the cylinder. On a 
more detailed note, it is interesting to see an inversion of $\Delta w$ when it gets close to the bottom. This is due to lubrication forces acting on the cylinder in the positive $z$-direction near the middle of the cylinder being larger than at its edges. It is easier for the liquid to escape the gap between the particle and the bottom near the edges than near the middle. We note that these are resolved lubrication forces: The inversion takes place well before the lubrication model mentioned previously kicks in.

Figure 5 shows that the simulation domain is sufficiently tall so as to have plateau (steady) values for $\operatorname{Re}_{s}$ and $\Delta w / d$ for a significant part of the trajectory. These plateau values we use for further analysis and verification purposes. This has been summarized in Figure 6 where steady state settling Reynolds number and deformation are plotted against $1 / \sigma$. The default spatial resolution $(d=12 \Delta$ for the LB method and $n_{s}=20$ for the number of segments along the cylinder) is compared to a finer LB grid and finer and coarser segment divisions. Steady state deformation is largely inversely proportional to the bending stiffness of the cylinder which is to be expected for the linear elastic behavior as implied by Eq. 4. Deviation from linearity for the most flexible cylinder (for which $1 / \sigma=2$ ) is the result of feedback from the fluid flow: The significant deformation that occurs when $1 / \sigma=2$ impacts the flow pattern around the cylinder which, as a consequence, steers the deformation away from the straight line that signifies linearity (Figure 6, bottom panel). The same feedback between deformation and flow results in a slight (note the limited $\left(\operatorname{Re}_{s}\right)_{s s}$ range of the upper panel of Figure 6) increase of settling speed with decreasing stiffness.

The numerical resolution has minor impact on the steady-state deformation and settling speed. A change in $n_{s}$ does - to a very good approximation - not affect the settling speed (and for that reason these data have been omitted in the upper panel of Figure 6). It does affect the deformation in a systematic manner: more segments results in increased deformation. However, differences between deformation for $n_{s}=20$ and 30 are less than $2 \%$. It should be noted that the simulation method sets some limitation on the number of segments. The length of each segment $\ell / n_{s}$ needs to be larger than $\Delta$ (by a factor of 2 at least) in order to have an appreciable number of marker points per segment to have a smooth and representative force distribution along the length of the cylinder. The LB resolution has some effect on the settling speed, as also 
seen in a previous paper on hindered settling [6]. Differences are, however, minor (less than 3\% between $d=12 \Delta$ and $d=16 \Delta)$.

\section{Hele-Shaw cell - comparison with experimental data}

As already noted in the Introduction, experiments under well-defined conditions with a complete set of material properties the results of which could be used for validation are rare. In this respect, the experimental part of the work by Wexler et al [16] on a single, clamped fiber deformed by the low-Reynolds number flow in a microfluidic channel is very useful. Their experimental results have been presented in the form of the fiber's tip deflection as a function of the volumetric flow rate in the channel. One of the foci of the experimental work is the effect of confinement as a result of which the channel width $W$ (see Figure $1 \mathrm{~b}$ ) is only slightly larger than the width of the fiber. An important detail of the experiment is that the fibers have a rectangular cross section, where our interest is in fibers with a circular cross section.

For a meaningful comparison between experiment and simulation we have translated the experimental data in dimensionless terms where the difference in cross sectional shape between simulation and experiment requires some caution. We define $w$ as the size of the fiber's cross section in streamwise direction and $s$ as its size in transverse direction. The fiber's length is (as before) denoted by $\ell$. Given the narrow confinement we give our simulated cylindrical fiber a diameter $d=s$ and make sure that in the simulations we match the experimental aspect ratios $s / W=0.85$ and $\ell / S=0.60$. The default length of the channel is $L=2 S$. In the flow direction we have periodic boundary conditions. Some cases have been repeated with $L=3 S$ and no significant differences with the default length were observed. The experimental bending parameter is defined as $\chi_{e x p}=\frac{\rho \nu U \ell^{2} s}{E I_{c s}}$ with $U=\frac{Q}{W S}$ and $Q$ the volumetric flow rate as specified in the experimental paper [16]. We realize that for the experimental fiber $I_{c s}=\frac{w^{3} s}{12}$. The rational for comparing experimental data with a specific value of $\chi_{\exp }$ with simulation data having the same value $\chi$ is that we expect the hydrodynamic force to scale with the superficial velocity and the frontal area which is $\ell d$ for the round fiber and $\ell s$ for the rectangular one so that the bending moment scales as $\ell^{2} d$ and $\ell^{2} s$ respectively. 
Figure 7 shows - in a double logarithmic way as also displayed in the experimental paper [16] - the comparison between experiment and simulation in dimensionless terms $\left(\chi_{\exp }\right.$ and $\chi$ versus the fiber tip deflection that has been scaled with the length of the fiber: $w_{3} / \ell$ ). The experimental data points have been obtained from the data set denoted " $w=22 \mu \mathrm{m}, h=241 \mu \mathrm{m}$ " in Figure 3 of Reference [16]. For this data set the aspect ratio of the fiber is $s / \ell=d / \ell=4.3$. The uncertainty in the experimental points in Figure 7 is due to the uncertainty in Young's modulus in the experiments that is reported as $E=63 \pm 22 \mathrm{kPa}$ [16]. The simulations have been conducted at a fixed flow rate and therefore fixed channel Reynolds number $\operatorname{Re}_{W}=U W / \nu \approx 0.1$. The bending parameter $\chi$ has been varied by varying $E I_{c s}$ (over almost three orders of magnitude). As in the experiments, the simulations show a linear and a non-linear regime with the transition occurring at $\chi \approx 10^{-2}$. The primary reason for the non-linear regime is feedback of the fiber deformation on the fluid flow and thus can - in principle - be captured by a linear elastic model for fiber bending. We observe reasonable agreement between simulation results and experimental data with, however, consistently lower deflection in the simulations. In the linear regime the deviations are within the error margin that is the result of uncertainty in Young's modulus in the experiment. This is not the case for the non-linear regime that has larger deformations. We here likely overstep the limit of small deformations $\left|w_{3}\right| \ll d$ that is part of the modeling approach. Also, and this is the case over the entire parameter range, there is the difference in cross sectional shape of the experimental and computational fiber with consequences for the hydrodynamic force distribution and therefore deflection. We do not expect, however, that this is an effect that has consequences beyond the error margins due to uncertainty in Young's modulus making it hard to assess conclusively.

There only are minor differences between simulation results with $d=16 \Delta$ and $24 \Delta$. On one hand, this is not surprising given the relatively simple flow geometry and low value of $\operatorname{Re}_{W}$. On the other hand, the narrow gap between fiber and channel side walls does require proper resolution. 
In conclusion, this test case of a fiber bending in micro channel flow has provided some confidence in the simulation approach. However, experimental validation under more challenging circumstances specifically involving assemblies of many particles - remains very desirable.

\section{Flexible cylinders in agitated flow}

The system we are looking into now is far more complicated than the ones in the previous sub-sections. It involves many flexible cylinders agitated by a non-trivial - albeit laminar - flow generated by a revolving impeller. This system is, however, a logical continuation of the simulations on agitated rigid cylinders discussed in [6] now using the methodologies for introducing flexibility as described (and to some level verified and validated) in the previous sections of the current paper. The main independent variable of this set of simulations is the bending stiffness. The solids volume fraction, Archimedes number, impeller-based Reynolds number and Shields number have all been kept constant. Most simulations have cylinders with $\ell / d=4$; one simulation has $\ell / d=6$. The initial condition is shown in Figure 8: random multilayers of cylinders on the bottom of the container, slightly bended under their own weight.

At time zero the impeller is inserted and set to rotate. In Figure 9 the evolution of what follows is monitored in terms of the average vertical $(z)$ position of the particles, as well as the average deformation. Differently from the one-cylinder settling and micro channel cases with deformation in one direction only, here deformation of particle $i \Delta w^{(i)}$ is defined as $\Delta w^{(i)}=\sqrt{\left(w_{2}^{(i)}(0)-w_{2}^{(i)}(\ell / 2)\right)^{2}+\left(w_{3}^{(i)}(0)-w_{3}^{(i)}(\ell / 2)\right)^{2}}$ and average deformation implies averaging over all particles. We observe a development towards a dynamic steady state over a period of at least 100 impeller revolutions. With respect to the average vertical particle position $\left\langle z_{p}\right\rangle$ there is no significant difference between the various $\ell / d=4$ cases. They all evolve towards a steady value of $\left\langle z_{p}\right\rangle \approx 0.36 H$. The longer $(\ell / d=6)$ particles get suspended slightly faster and - on average - to a slightly higher vertical level. This trend with aspect ratio was also observed for rigid cylinders [6]. Average deformation initially develops quickly. Steady state values - obviously - depend on the stiffness parameter $\sigma$. 
Impressions of the solids suspension process are given in Figure 10. The upper row is a time sequence with initially particles being drawn to the impeller from underneath due to the pressure distribution induced by the swirling flow with minimum pressure under the impeller. Particles can be seen to be strongly deformed by the impeller in Figure 10b. The dynamic steady state is one characterized by partial suspension (Figure 10c) as anticipated by the Shields number value of $\theta=9.08$ [30] and the average vertical particle location being well below $H / 2$ (see Figure 9). The impressions in the lower row of panels of Figure 10 clearly illustrate the effect of bending stiffness and cylinder length on the levels of deformation of the particles.

Partial suspension of particles is also witnessed from the vertical solids volume fraction profiles in Figure 11. Layering of particles is observed closely above the bottom. There are no clear, systematic differences between the profiles except for the second layering peak above the bottom being much weaker for $\ell / d=6$ which indicate that these longer particles are suspended better, and the fact that the minimum $\phi$ at the level of the impeller $(z \approx H / 2)$ is less pronounced for the most flexible particles with $\ell / d=4$ as well as for the particles with $\ell / d=6$.

In Figure 12 we focus on the way deformation of particles is distributed. In the top panel as probability distribution functions (pdf's) which have been collected over the entire tank volume as well as over a time period of 25 impeller revolutions; in the bottom panel as the average deformation as a function of vertical position of the particle centers. From the pdf's it is clear that many particles deform strongly and have deformations $\Delta w$ of the order of the particle diameter $d$. The fraction of particles deforming strongly increases more than proportionally with decreasing $\sigma$, with $\sigma$ varying by 1 to 2 orders of magnitude the variations in the pdf's exceed 2 orders of magnitude. The average level of deformation is clearly coupled with local flow conditions: it peaks at the height of the impeller, more precisely directly above and directly below the impeller. With deformation of the order of particle diameter $d$, extending the simulation procedure beyond the small deformation limit $\Delta w \ll d$ is an important next step. The results in Figure 12 show that specifically if $\sigma<50(\ell / d=4)-$ this condition is not satisfied. 
The two panels of Figure 12 indicate that the deformation levels of the simulation with $\ell / d=4 \& \sigma=5$ and the one with $\ell / d=6 \& \sigma=50$ are comparable, as also seen in the time series in Figure 9. Deflection of a linear elastic beam subjected to a certain load is proportional to $\ell^{4}$ [28]. With $(6 / 4)^{4} \approx 5$ we thus expect comparable deformations for the two cases. As we observed in Figure 11, the two cases showed a slightly higher solids volume fractions at the level of the impeller hinting that (strong) flexibility has consequences for the way particles interact with the impeller.

In an overall sense, the interactions between impeller and particles and between impeller and fluid have been quantified by measuring the torque exerted on the impeller. In the conventional way of mixing research [31], torque has been translated into power $P$ first and then into the dimensionless power number Po: $P=2 \pi N \Gamma$ and $\mathrm{Po}=P /\left(\rho N^{3} D^{5}\right)$ with $\Gamma$ the torque exerted on the impeller. The torque, and therefore Po, can be divided in a fluid and a solids contribution. The fluid contribution follows from integrating the fluid-impeller interaction forces stemming from the IBM over the impeller surface, the solids contribution from integrating the contact forces between impeller and the particles. Time series of Po are given in Figure 13 for the case with the most flexible particles $(\sigma=5)$ of aspect ratio $\ell / d=4$ and for a case with the same aspect ratio but much higher stiffness $(\sigma=50)$. The two cases behave very differently. All four time series in Figure 13 fluctuate which is due to particle motion, not due to turbulence given the modest impeller-based Reynolds number. The interaction between solids and impeller for $\sigma=5$ is very weak which we interpret as the flexibility of the particles enabling them to largely avoid contact with the impeller. This is very different for the $\sigma=50$ particles. The torque fluctuations due to these particles are much higher indicating many more particle-impeller collisions. These differences in particle behavior of relatively stiff and flexible particles has profound consequences for the fluid-related torque which is much higher for the flexible particles.

Time-averaged power number results are shown in Figure 14. The total power (fluid plus solids power) for $\ell / d=4$ decreases with $\sigma$ for $\sigma<50$ and for $\sigma \geq 50$ gets independent of the stiffness parameter. The single data point for $\ell / d=6$ shows - as also seen in Figure 12 - results comparable to the case with 
$\ell / d=4 \& \sigma=5$. The total torque (or total power) is a measurable quantity. The simulations suggest that it depends on the flexibility of the particles if they are sufficiently flexible. An experiment along those lines would be an interesting test for the simulations presented in this paper.

The time series on power / torque in Figure 13 provide us with some insight in the time scales of fluctuations induced by the revolving impeller as well as by particle motion. An order of magnitude estimate of the fluctuation time scale based on what we see in Figure 13 is $\tau_{\text {flow }} \approx 0.1 / N$ which is equivalent to 400 time steps $(400 \Delta t)$. The time scale of bending dynamics is estimated as $\tau_{\text {bend }}=1 / f_{\text {bend }}=\sqrt{\frac{\ell^{3} m}{E I}}$ which is for a mid-range stiffness coefficient of $\sigma=50$ - of the same order of magnitude as the flow time scale, casting into some doubt the validity of the quasi-static assumption underpinning Eq. 3.

\section{Summary and conclusions}

A methodology for highly resolved flow simulations of flexible cylindrical particles suspended in liquid has been introduced. It is an extension of previous work on particle-resolved simulations with rigid cylinders. Based on the way the hydrodynamic forces and particle interaction forces are distributed along the length of the cylinders, bending moments and subsequently deflections have been calculated based on linear elastic theory and a quasi-static assumption.

For the relatively simple case of a cylinder settling horizontally in a closed container at low Reynolds number a series of verification tests have been performed. They show only a weak dependency on the numerical parameters of the settling speed and the level of bending of the particle. It is also clear from these simulations that the particle deformation is fed back to the fluid flow and that the settling speed increases albeit modestly - with increasing deformation. We also have reproduced - with a fair level of agreement experimental results on bending of a clamped fiber in micro channel flow [16], including the transition from a linear to a non-linear regime which is the result of feedback of the fiber shape on the flow in the channel and drag force on the fiber. 
To demonstrate the feasibility of the numerical approach to many particle systems in complex flow, flexible cylinders were placed in an agitated tank to an overall solids volume fraction of 0.15 . The agitation was such that a laminar flow with an impeller-based Reynolds number of 87 was generated. We mainly investigated how the system responds to a change in the bending stiffness of the cylinders. In the flexibility range considered, the deflection of the $\ell / d=4$ cylinders is of the order of the diameter $d$ of the cylinders with deflection being on average strongest near the impeller. The levels of solids suspension as measured through the time-averaged vertical location of the particles and the vertical solids volume fraction profile do not depend strongly on the flexibility of the particles.

The torque required to spin the impeller and the way it is distributed over a fluid and a solids contribution does depend significantly on the bending stiffness. The resulting total power number is higher when the particles are more flexible and then is almost completely due to the fluid. For more rigid particles a significant portion of the torque is required for direct particle-impeller interactions.

We have shown that the assumptions of small deformation and quasi-static deflection are not fully satisfied under many of the conditions investigated in this paper. Refining the simulation procedure to alleviate restrictions on the applicability of the computational method is left for future work. We do not expect conceptual issues in this respect. Including dynamics in the bending process to overcome the quasisteady assumption requires adding time-dependent terms in the structural equations where accurate time stepping needs careful attention. Also theory for large deflections - still based on linear elastic material - is well developed [28] and amenable for implementation in numerical procedures. Inspiration can also be obtained from research on fluid-structure interaction (FSI) where solid and fluid mechanics solvers are coupled to study large deformation of structures under the influence of fluid flow over a very wide range of Reynolds numbers, from creeping flow [32], to intermediate [33] to high Reynolds numbers [34].

In light of this, however, it is (more) important to further work on experimental validation in order to test the simulation procedure and see if refinements in the procedure have sizeable effects in the right direction. It is realized, however, that creating the flow systems experimentally with particle of the very specific properties and dimensions we have been investigating is not an easy task. 
An extension in a different direction is assessment of particle breakage probabilities [35]. Given that we now have a method to determine the mechanical load on cylindrical particles in complex flow of dense suspensions, we can assess probability of breakage of cylinders having a certain strength. The simulations could actually perform the breakage event and - starting from a certain cylinder length (distribution) - make predictions of a resulting length distribution as a function of agitation conditions.

\section{References}

[1] Mallick PK. Fiber reinforced composites, materials, manufacturing and design, third edition. Boca Raton: CRC Press; 2007.

[2] Chen D, Jiang K, Huang T, Shen G. Recent advances in fiber supercapacitors: materials, device configurations, and applications. Advanced Materials 2019; in print: doi: 10.1002/adma.201901806.

[3] Dionisi D, Anderson JA, Aulenta F, McCue A, Paton G. The potential of microbial processes for lignocellulosic biomass conversion to ethanol: a review. Journal of Chemical Technology \& Biotechnology 2015; 90: 366-383.

[4] Xi Y, Chen Q, You C. Flow characteristics of biomass particles in a horizontal stirred bed reactor: Part I. Experimental measurements of residence time distribution. Powder Technology 2015; 269: $577-584$.

[5] Derksen JJ. Liquid fluidization with cylindrical particles: Highly resolved simulations. AIChE Journal 2019; 65: pe16594-1-11.

[6] Derksen JJ. Agitating cylindrical particles in laminar liquid flow. Chemical Engineering Research and Design 2019; 154: 11-20.

[7] Kempe T, Fröhlich J. An improved immersed boundary method with direct forcing for the simulation of particle laden flows. Journal of Computational Physics 2012; 231: 3663-3684.

[8] Wachs A, Hammouti A, Vinay G, Rahmani M. Accuracy of finite volume/staggered grid distributed Lagrange multiplier/fictitious domain simulations of particulate flows. Computers \& Fluids 2015; 115: 154-172.

[9] Wachs A. Particle-scale computational approaches to model dry and saturated granular flows of nonBrownian, non-cohesive, and non-spherical rigid bodies. Acta Mechanica 2019; 230: 1919-1980.

[10] Wu J, Aidun CK. Simulating 3D deformable particle suspensions using lattice Boltzmann method with discrete external boundary force. International Journal of Numerical Methods in Fluids 2010; 62:765-783.

[11] du Roure O, Lindner A, Nazockdast EN, Shelley MJ. Dynamics of flexible fibers in viscous flows and fluids. Annual Review of Fluid Mechanics 2019; 51:539-72. 
[12] Qi D. A new method for direct simulations of flexible filament suspensions in non-zero Reynolds number flows. International Journal of Numerical Methods in Fluids 2007; 54:103-118.

[13] Derksen JJ. Direct meso-scale simulations of fibers in turbulent liquid flow. Canadian Journal of Chemical Engineering 2010; 88, 677 - 681.

[14] Delmotte B, Climent E, Plouraboué F. A general formulation of Bead Models applied to flexible fibers and active filaments at low Reynolds number. Journal of Computational Physics 2015; 286:14-37.

[15] Wiens JK, Stockie JM. Simulating flexible fiber suspensions using a scalable immersed boundary algorithm. Computational Methods Applied Mechanical Engineering 2015; 290:1-18

[16] Wexler JS, Trinh PH, Berthet H, Quennouz N, du Roure O, Huppert HE, Lindner A, Stone HA. Bending of elastic fibres in viscous flows: the influence of confinement. Journal of Fluid Mechanics 2013; 720:517-44

[17] Chen S, Doolen GD. Lattice-Boltzmann method for fluid flows. Annual Review of Fluid Mechanics 1998; 30: 329-364.

[18] Aidun C, Clausen J. Lattice-Boltzmann method for complex flows. Annual Review of Fluid Mechanics 2010; 42: $439-472$.

[19] Kruger T, Kusumaatmaja H, Kuzmin A, Shardt O, Siilva S, Viggen EM. The lattice Boltzmann method: Principle and practice. Springer; 2017.

[20] Somers JA. Direct simulation of fluid flow with cellular automata and the lattice-Boltzmann equation. App. Sci. Res. 1993;51: 127-133.

[21] Eggels JGM, Somers JA. Numerical simulation of free convective flow using the lattice-Boltzmann scheme. Int. J. Heat Fluid Flow 1995; 16: 357-364.

[22] Ten Cate A, Nieuwstad CH, Derksen JJ, Van den Akker HEA. PIV experiments and lattice-Boltzmann simulations on a single sphere settling under gravity. Phys. Fluids 2002; 14: 4012-4025.

[23] Williams JR, O'Connor R. Discrete element simulation and the contact problem. Archives of Computational Methods in Engineering 1999; 6: 279-304.

[24] Kuipers JB. Quaternions and Rotation Sequences. Princeton: Princeton University Press; 1999.

[25] Goldstein H. Classical mechanics (second edition). Reading, Massachusetts: Addison-Wesley, 1980.

[26] Phillips WF. Review of attitude representation used for aircraft kinematics. J. Aircraft 2001; 38: 718737.

[27] Suzuki K, Inamuro T. Effect of internal mass in the simulation of a moving body by the immersed boundary method. Comp. \& Fluids 2011; 49: 173-187.

[28] Timoshenko SP, Gere JM. Mechanics of materials. New York: Van Nostrand Reinhold; 1973.

[29] Blevins R. Formulas for Natural Frequency and Mode Shape. Malabar, Florida: Krieger; 1979.

[30] Derksen JJ. Highly resolved simulations of solids suspension in a small mixing tank. AIChE Journal 2012; 58: $3266-3278$. 
[31] Hoogendoorn CJ, Den Harttog AP. Model studies on mixers in the viscous flow region. Chemical Engineering Science 1967; 22: 1689-1699.

[32] Manikantan H, Saintillan D.. Manikantan H, Saintillan D. Buckling transition of a semiflexible filament in extensional flow. Phys. Rev. E 2015; 92: 041002.

[33] Sahu TR, Furquan M, Mittal S. Numerical study of flow-induced vibration of a circular cylinder with attached flexible splitter plate at low Re. J. Fluid Mech. 2019; 880: 551-593.

[34] Gurugubelli PS, Jaiman RK. Large amplitude flapping of an inverted elastic foil in uniform flow with spanwise periodicity. J. Fluids Structures 2019; 90: 139-163.

[35] Schöngut M, Grof Z, Štěpánek F. Analysis of breakage patterns in a sheared layer of elongated particles. Powder Technology 2019; 345: 682-691.

\section{Figures}

Figure 1. Flow configurations. (a) Top and side view of the single cylinder (with diameter $d$ and length $\ell$ ) settling in a fully closed container of dimensions $8 d \times 8 d \times 12 d$; (b) side and front view of micro channel with a cylinder clamped to the bottom; (c) top and side view of the stirred tank with down-pumping pitchedblade turbine. Cartesian coordinate systems as indicated. Gravity is pointing in the negative $z$-direction for the settling system (a) and mixing tank (c).
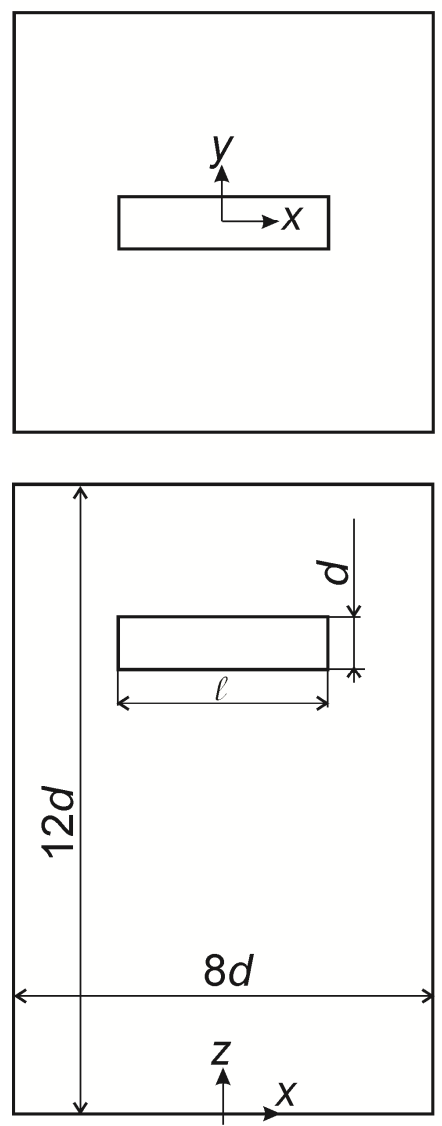

(a)
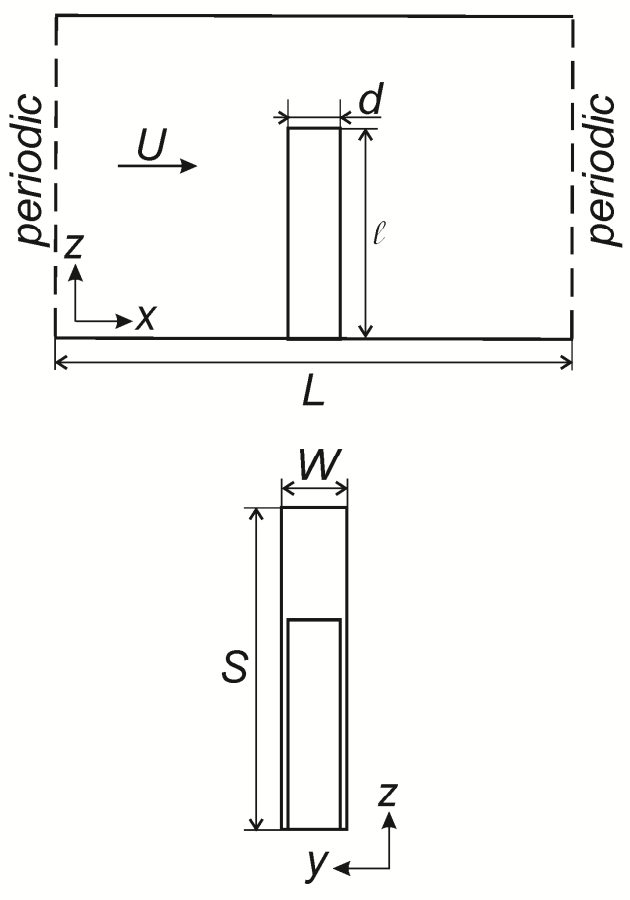

(b)
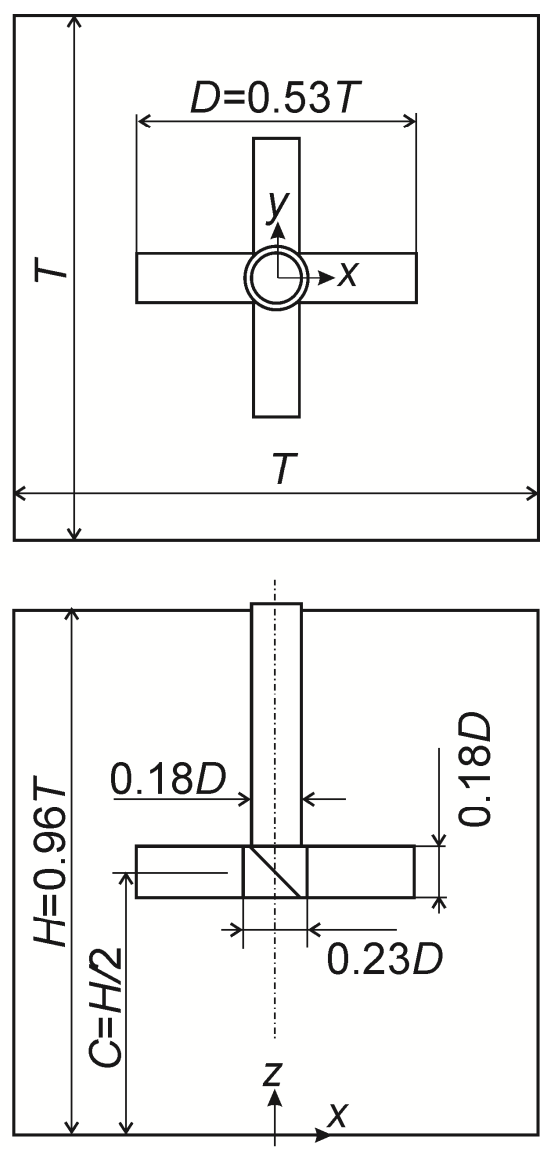

(c) 
Figure 2. The $\left(x_{1}, x_{2}, x_{3}\right)$ coordinate system attached to each cylinder. The load (force per unit length) distributions responsible for bending are indicated by $a_{2}$ and $a_{3}$.

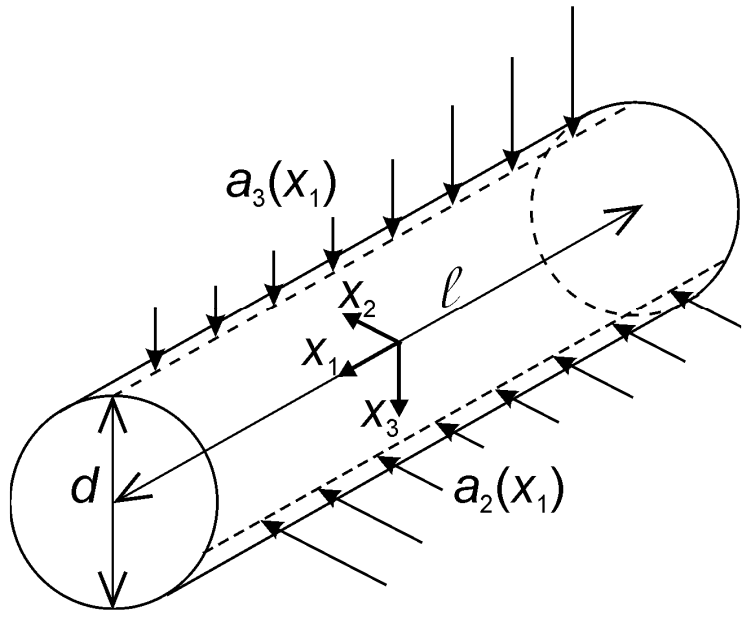

Figure 3. (a) Undeformed (black) and deformed (red) centerline of the cylinder with segments and nodes (the dots in the middle of each segment); (b) marker points on an undeformed cylinder, the alternating red and blue color indicates the segments; (c) marker points on a deformed cylinder.

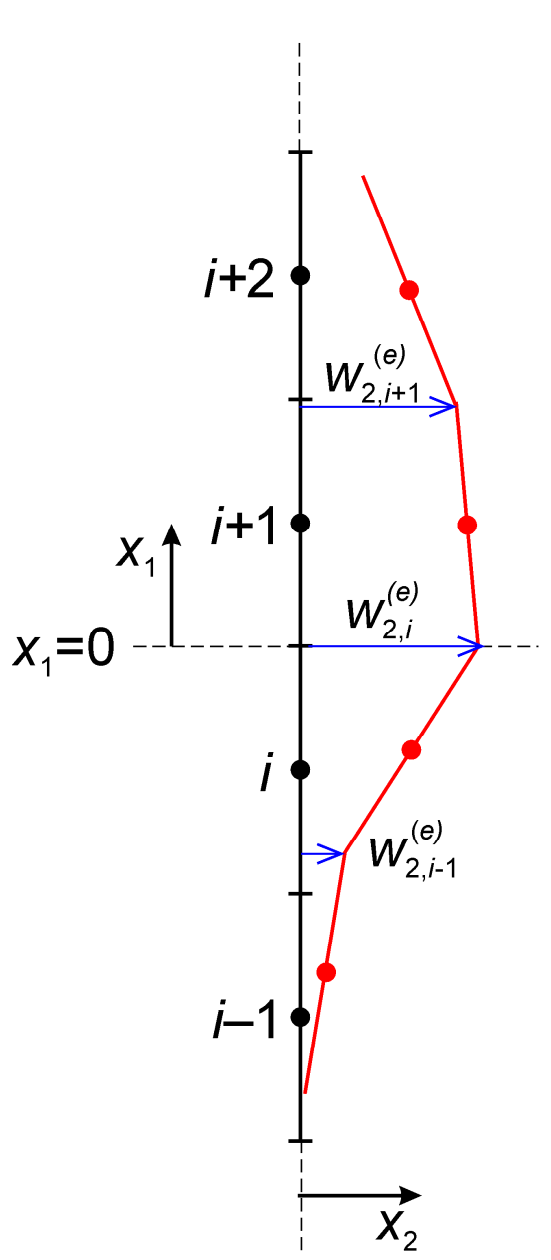

(a)

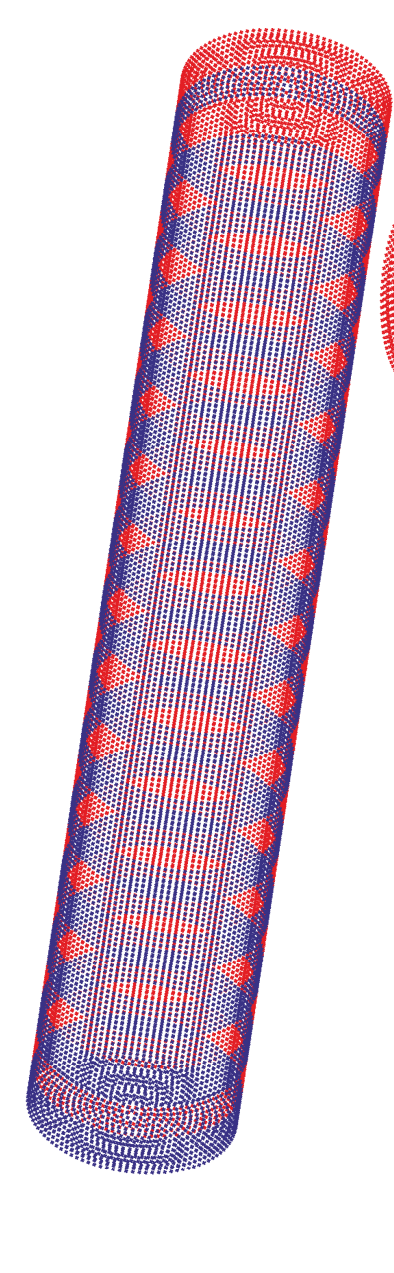

(b)

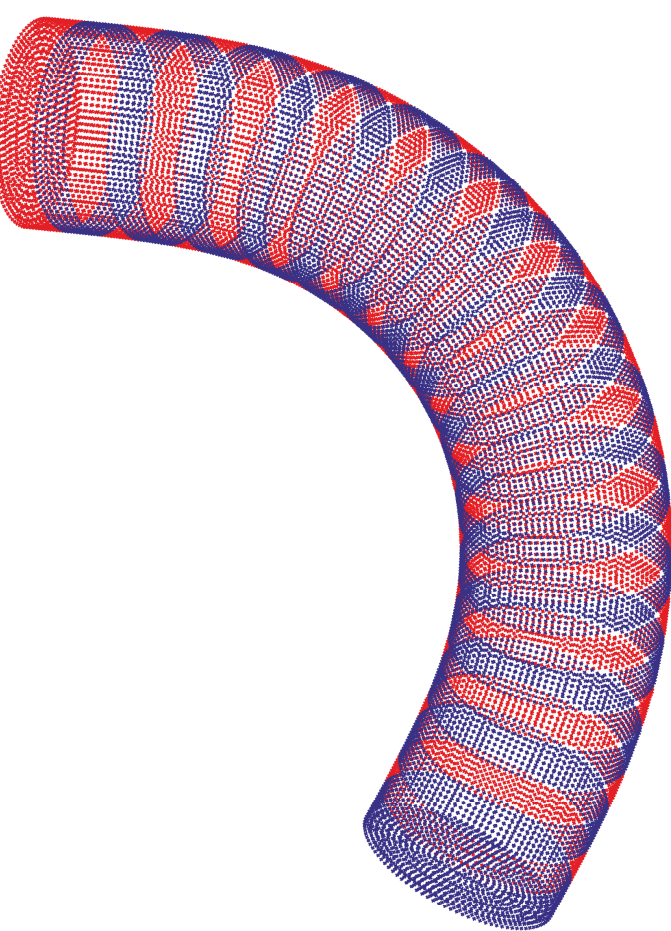

(c) 
Figure 4. Cylinder with $\ell / d=4$ and $\sigma=0.5$ settling in a closed box. Three time instants as indicated.
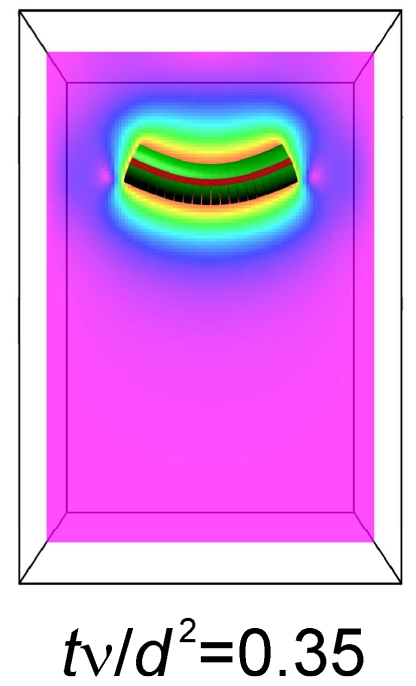

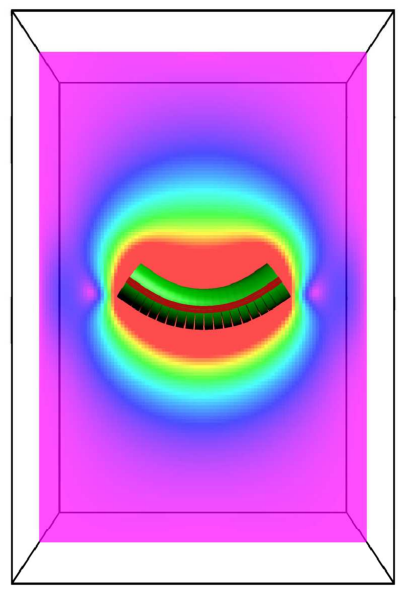

6.9

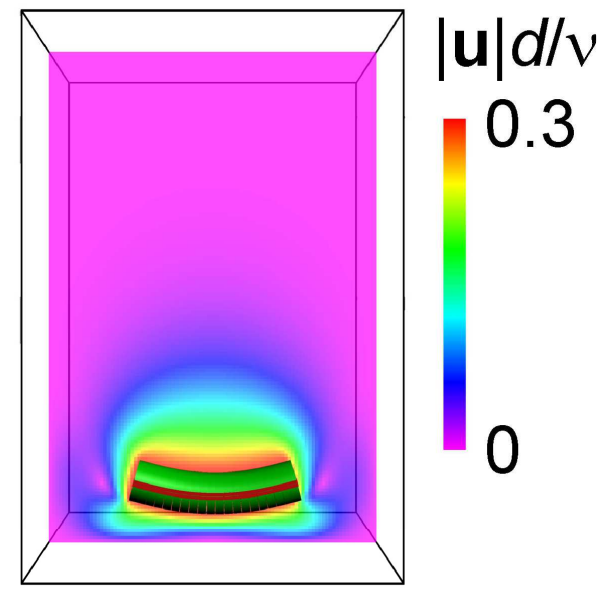

17

Figure 5. Time series of the Reynolds number of a settling cylinder (top) and its deflection (bottom) with $\Delta w$ defined in the text. Cylinder with $\ell / d=4$ and stiffness parameter $\sigma$ as indicated.
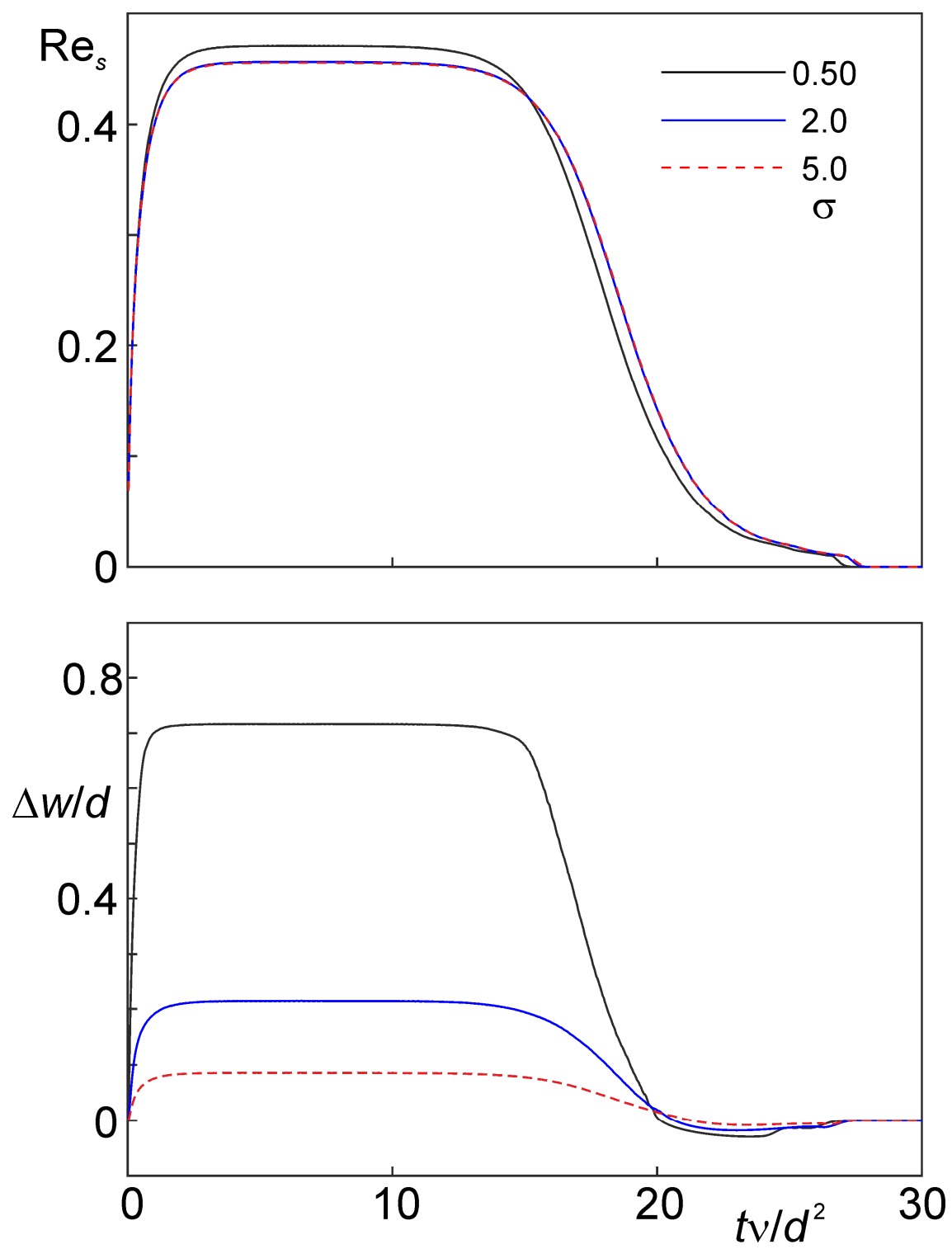
Figure 6 The plateau settling Reynolds number $\left(\operatorname{Re}_{s}\right)_{s s}$ and deflection $(\Delta w)_{s s}$ as a function of $1 / \sigma$. Comparing different resolutions for the number of segments $n_{s}$ and the number of lattice cells per diameter $d$. The dashed line in the lower panel is to indicate the extent of linearity.

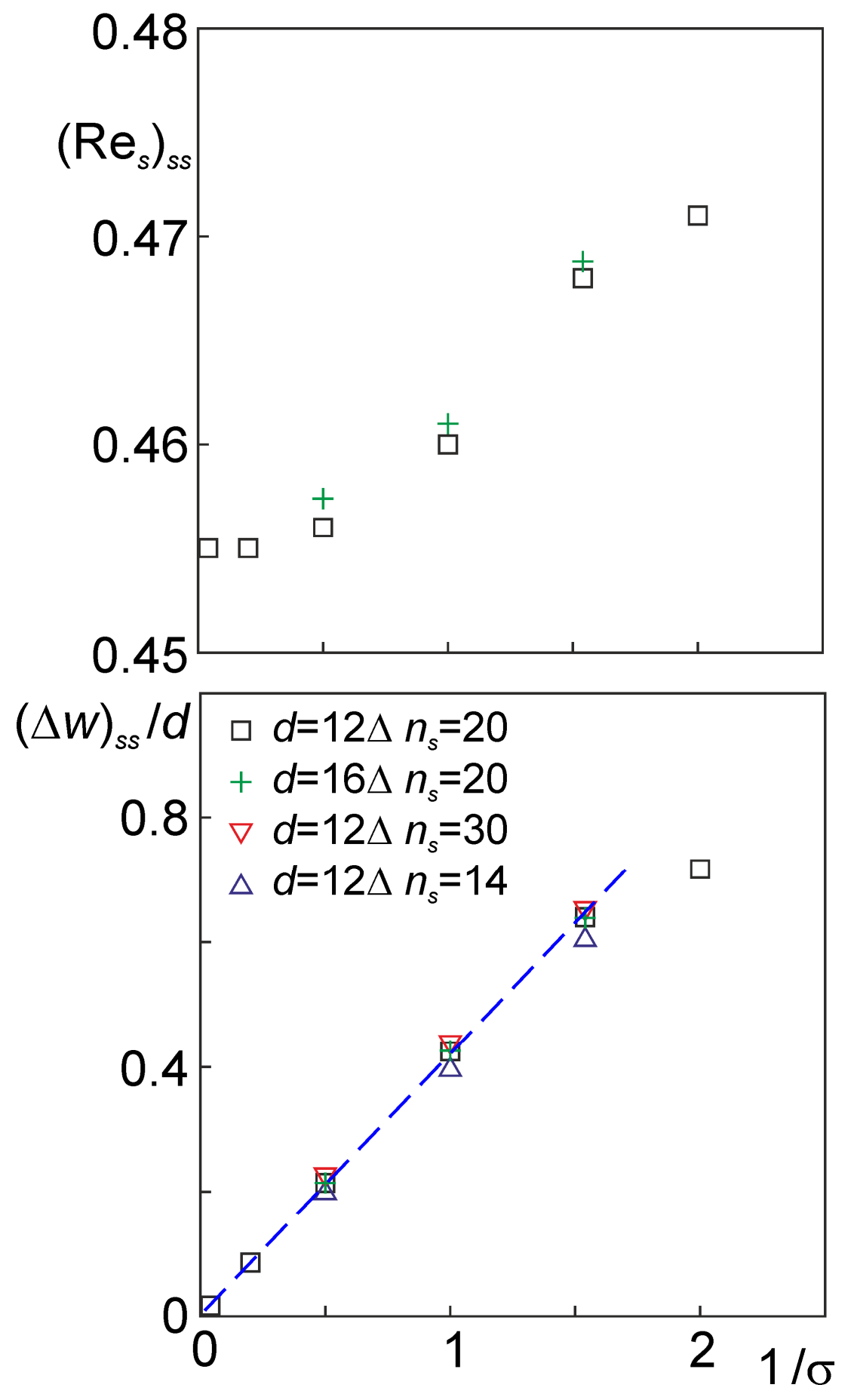


Figure 7. Clamped cylinder bending in micro channel flow: tip deflection versus bending parameter $\chi$ (defined in the text). Comparison between experimental results due Wexler et al [16] and simulations at two resolutions as indicated. The inset is a side view of the simulation related to the filled symbol with colors representing velocity magnitude in the mid plane. The uncertainty in the experimental data - as indicated in one of the symbols - is due to a $30 \%$ uncertainty in Young's modulus.

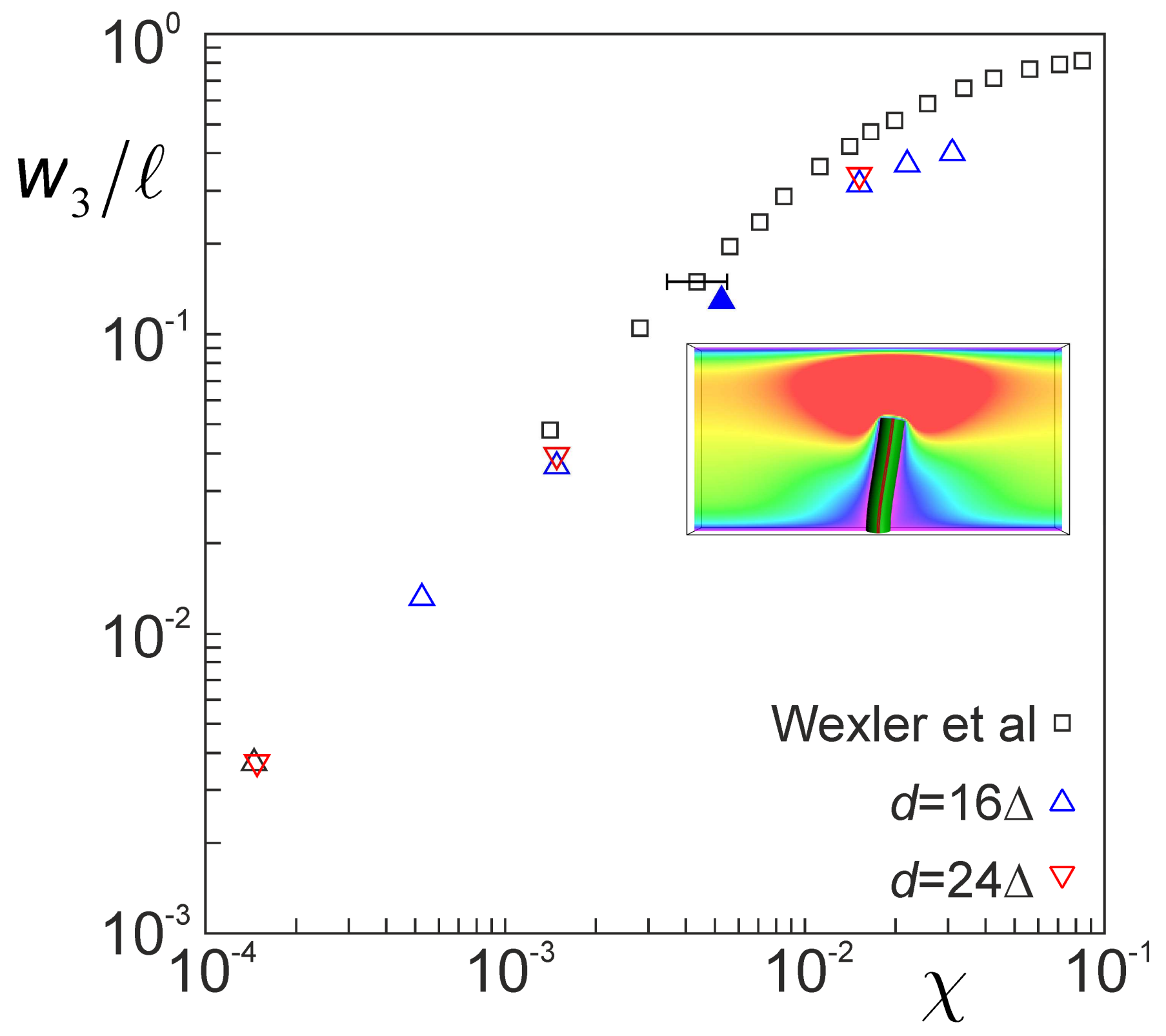


Figure 8. Initial location of cylinders after having settled. Left: $\ell / d=4$ and $\sigma=15$; right $\ell / d=6$ and $\sigma=50$.
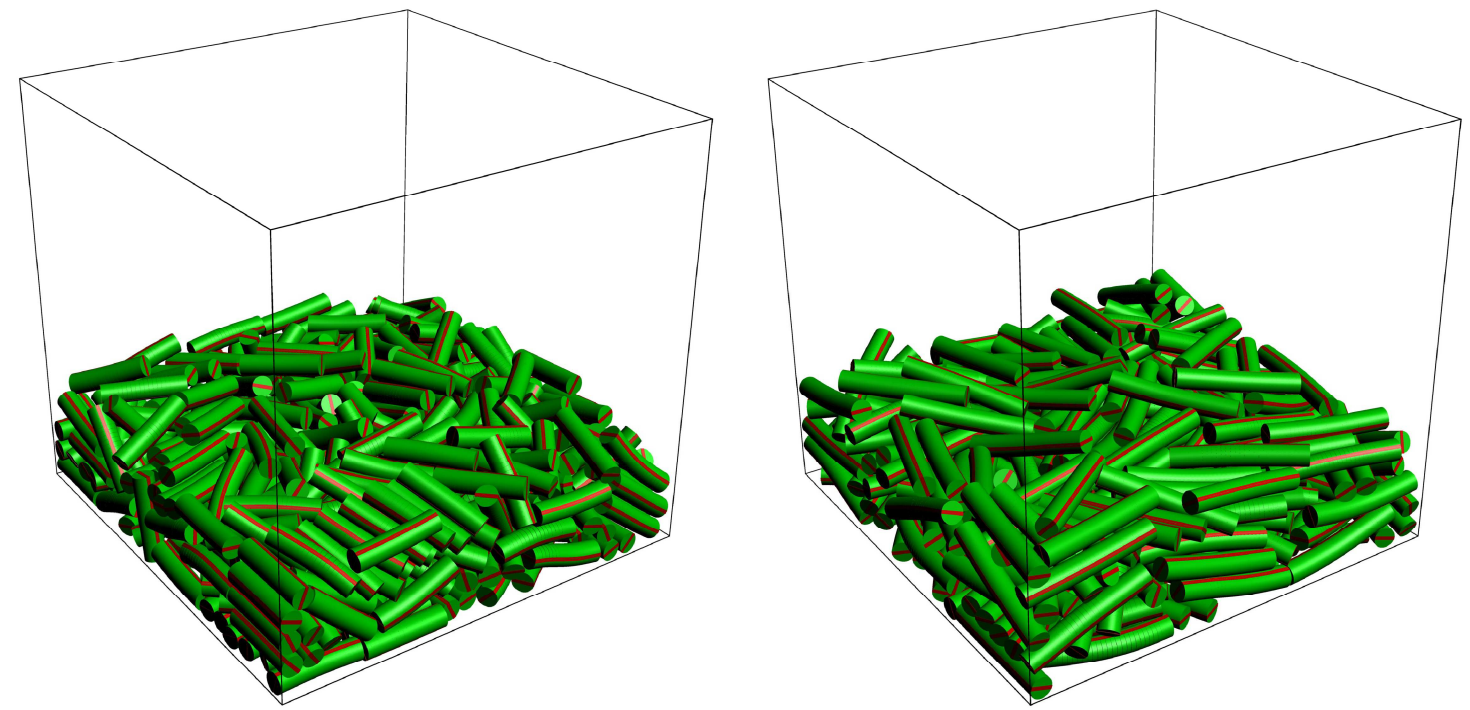

Figure 9. Time series of the average vertical location of the centers of the particles (top) and average deformation of the particles (bottom). Particle stiffness and aspect ratio as indicated. Loss of data is causing a gap in results for the case $\ell / d=4 \& \sigma=150$, as well as the case $\ell / d=6 \& \sigma=50$ not starting from $t N=0$.
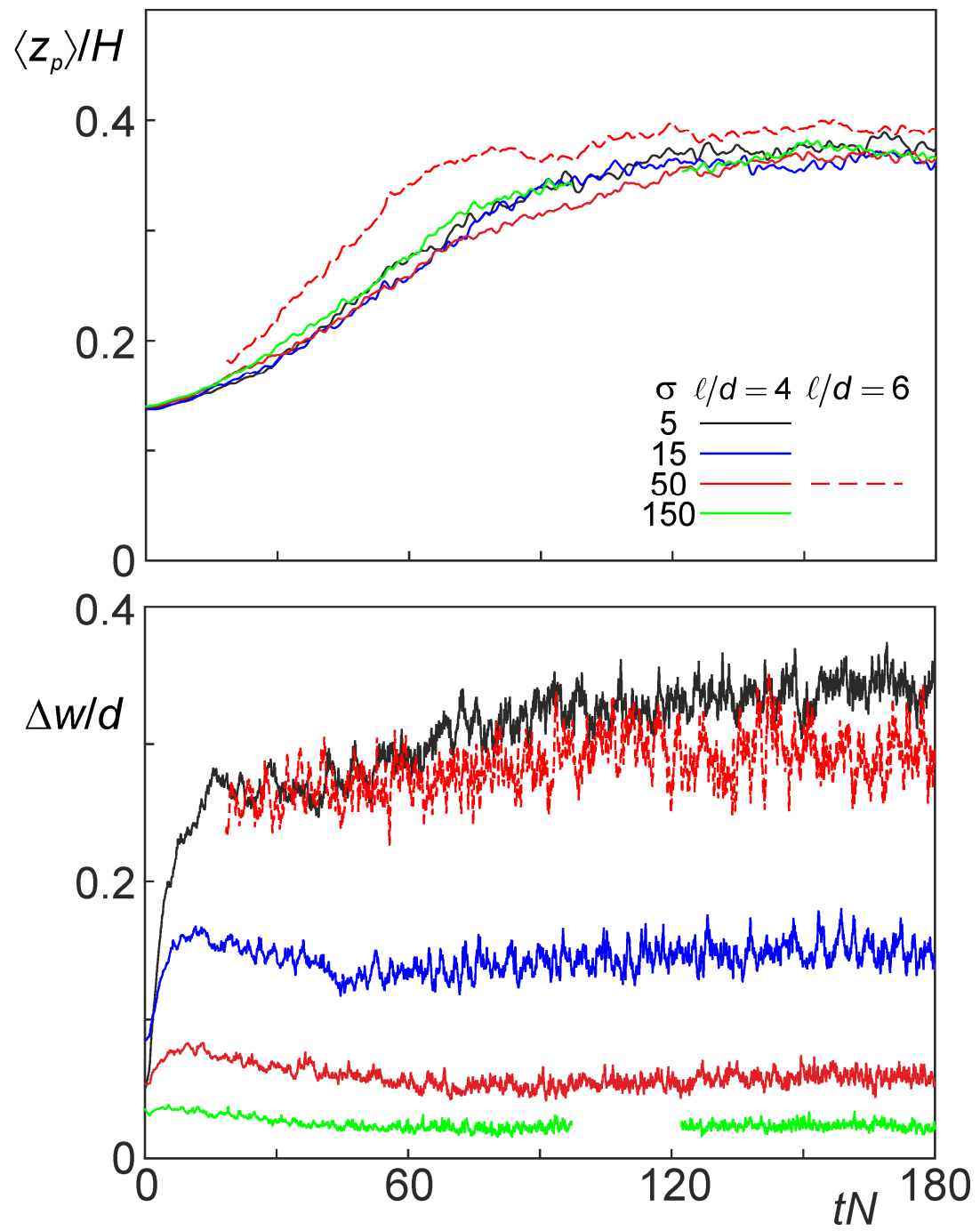
Figure 10. Impressions of particle suspension along with velocity magnitude contours in the mid-plane. The top row shows the evolution of the system with $\ell / d=4$ and $\sigma=5.0$ from start-up at moments $t N=14.4$ (a), 30.0 (b) and 130 (c). Bottom row, snapshots of other systems after reaching quasi steady state. (d): $\ell / d=4$ and $\sigma=15.0$; (e) $\ell / d=4$ and $\sigma=50.0$; (f) $\ell / d=6$ and $\sigma=50.0$.

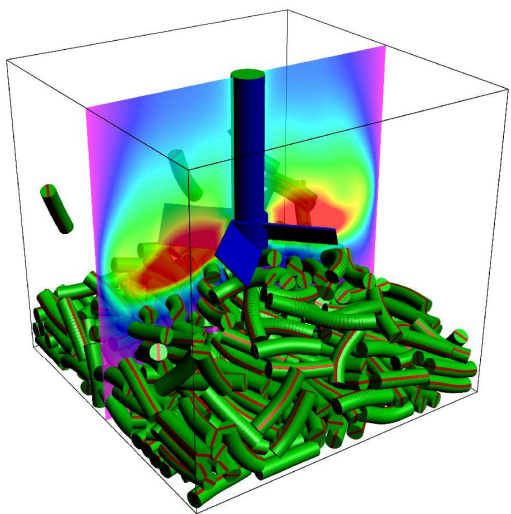

(a)

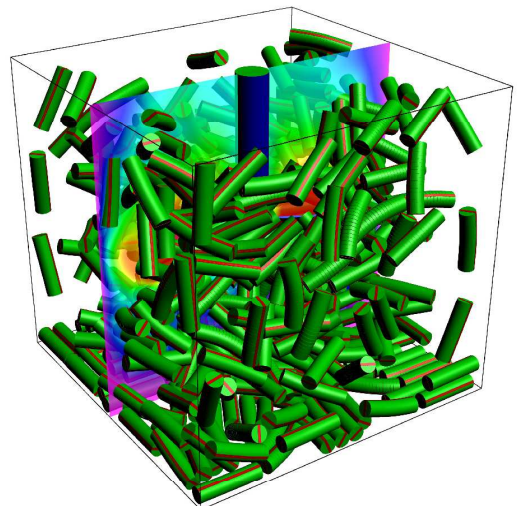

(d)

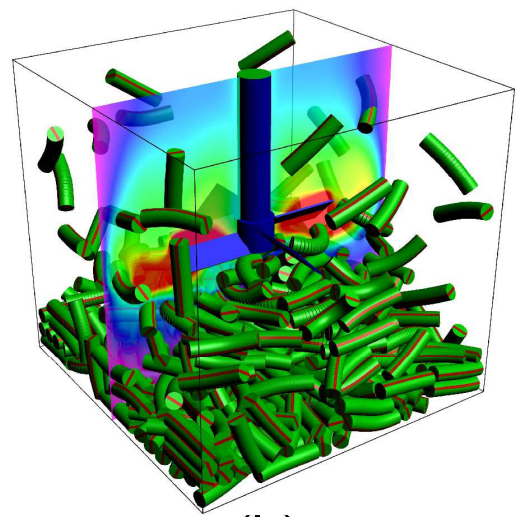

(b)

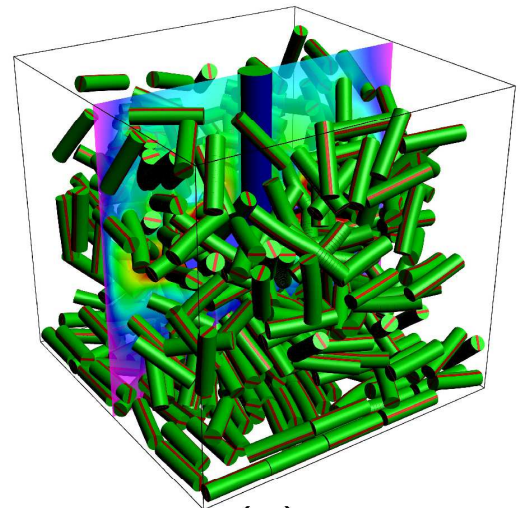

(e)

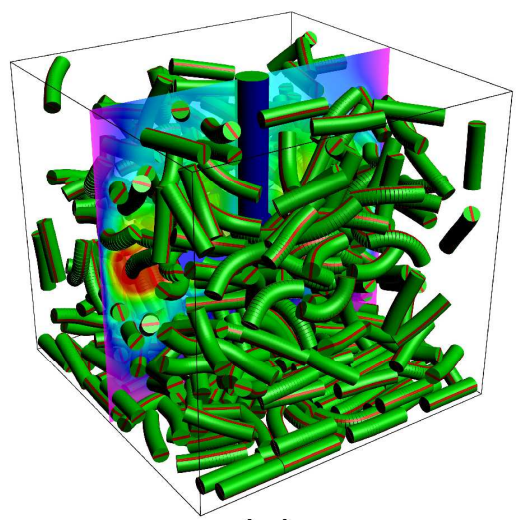

(c)

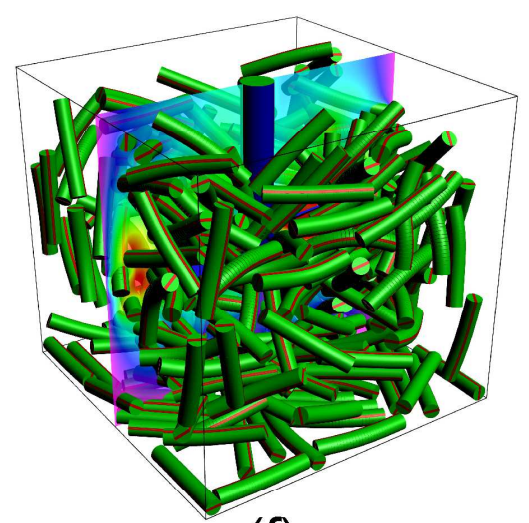

0.5 (f)

$u / V_{\text {tip }}$ 
Figure 11. Time-averaged solids volume fraction after steady state has been reached as a function of the vertical location in the tank. Four cases with $\ell / d=4$, one case with $\ell / d=6$. Stiffness parameter $\sigma$ as indicated.

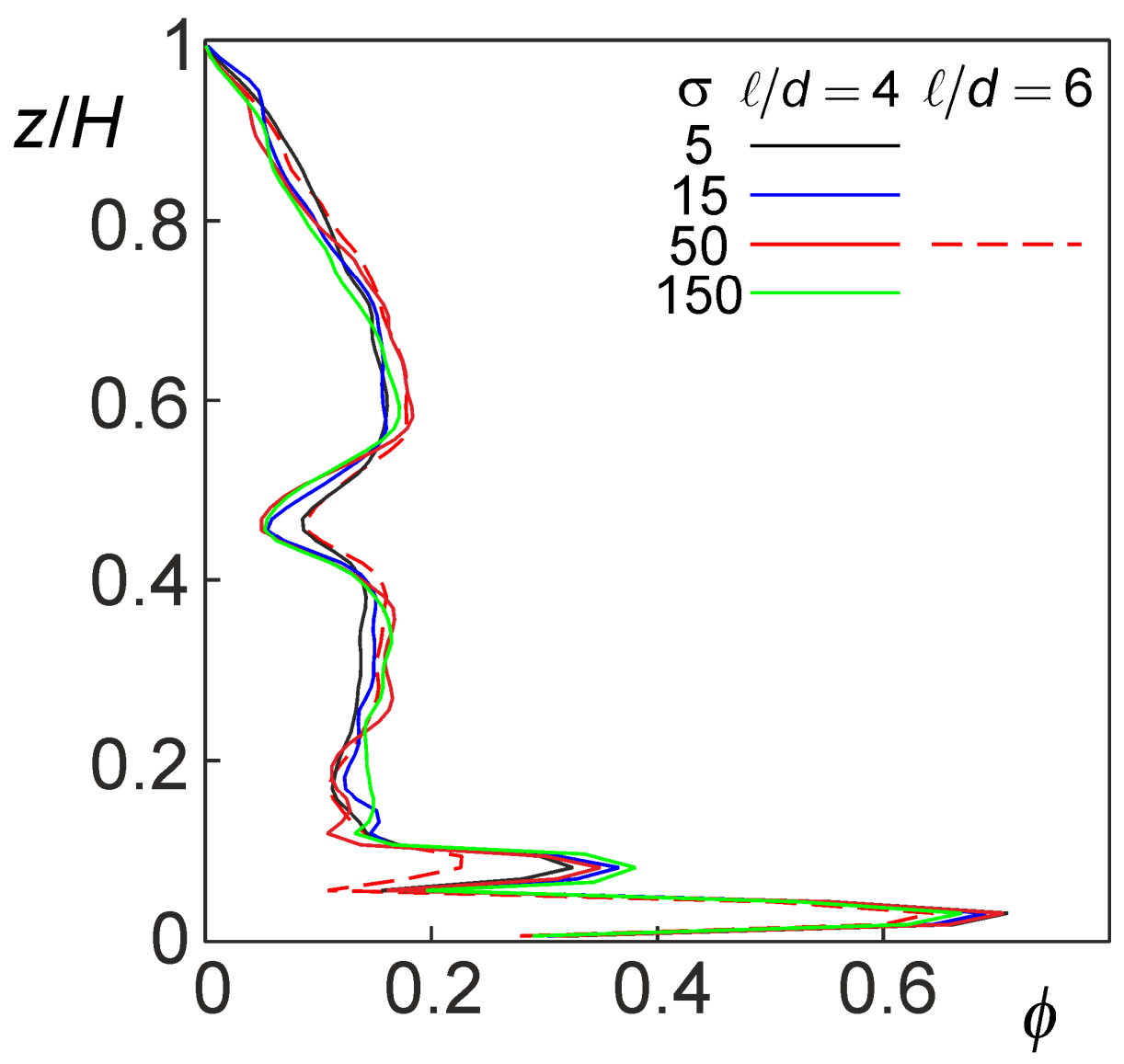


Figure 12. Top: probability distribution function (pdf) of particle deformation $\Delta w / d$ for various stiffness coefficients $\sigma$. Bottom: average distribution of particle deformation as a function of the vertical location. Cylinders with $\ell / d=4$ and one case with $\ell / d=6$, time-averaging over 25 revolutions after dynamic steady state has been reached.
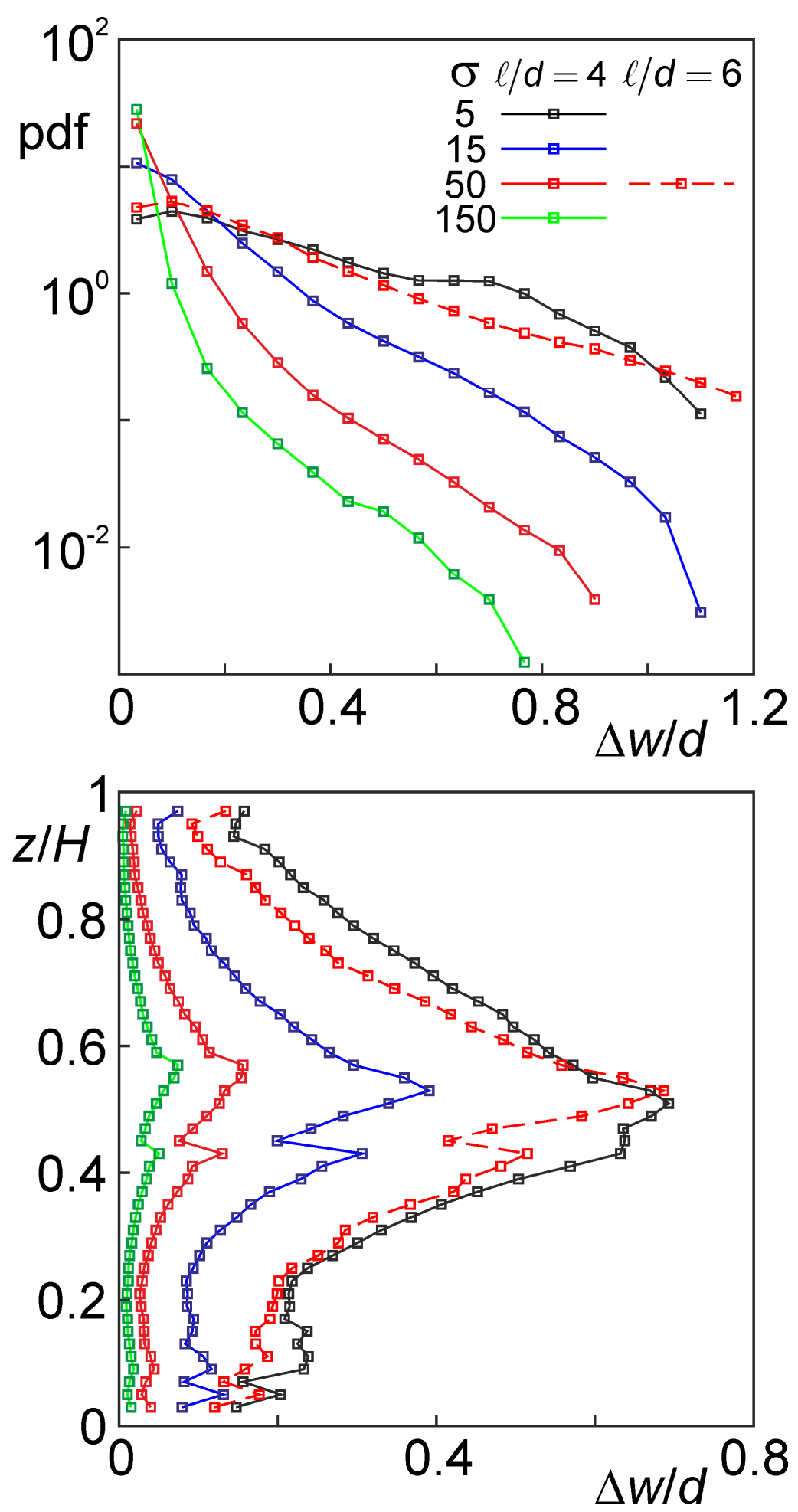
Figure 13. Time series - after a dynamic steady state has been reached - of the torque (translated in a power number Po, see text) required to revolve the impeller with contributions from fluid and solids as indicated for simulations with $\sigma=5$ and 50 .

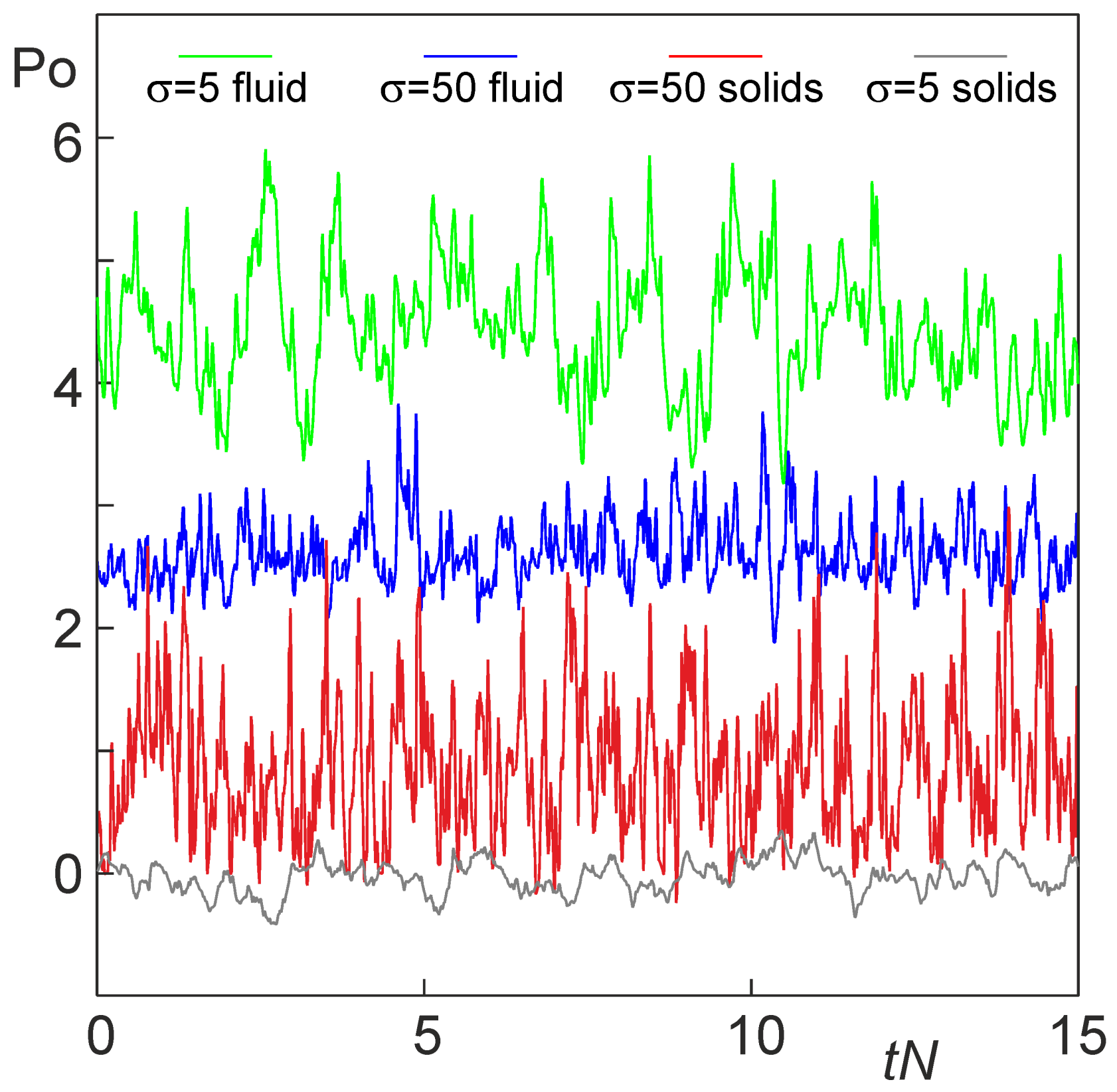


Figure 14. Time-averaged power number Po as a function of $\sigma$ for $\ell / d=4$ (open symbols) and $\ell / d=6$ (closed symbols). Total power is the sum of fluid power and solids power.

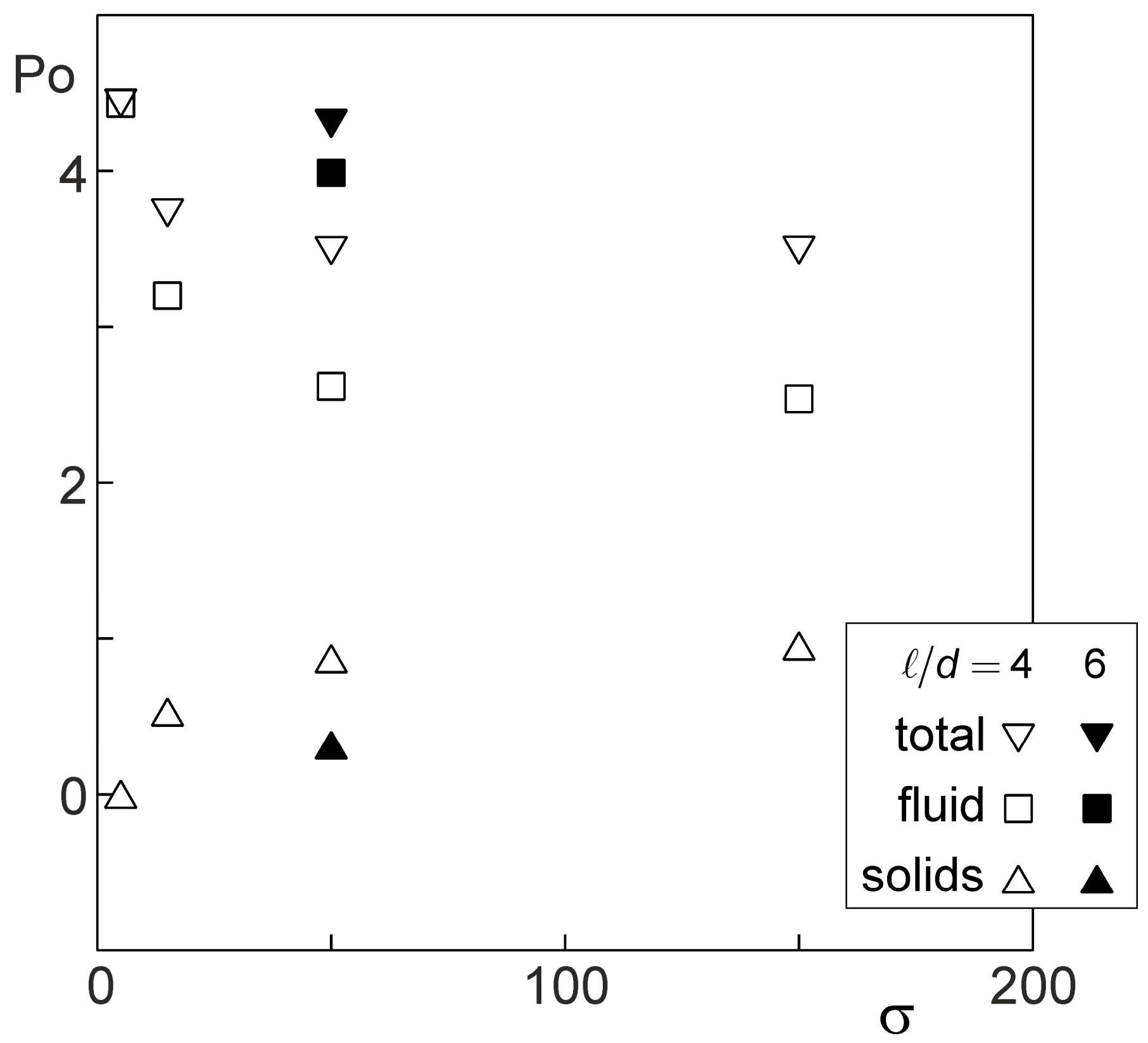


Tables

Table 1. Definitions and settings of dimensionless numbers

\begin{tabular}{|l|l|l|}
\hline Expression & Value or range & Description \\
\hline $\mathrm{Ar}=(\gamma-1) g d^{3} / \nu^{2}$ & 4.15 & Archimedes number \\
\hline $\mathrm{Re}=N D^{2} / \nu$ & 87 & Impeller Reynolds number \\
\hline $\operatorname{Re}_{s}=u_{s} d / \nu$ & dependent variable & Settling Reynolds number \\
\hline $\operatorname{Re}_{W}=U W / \nu$ & $\approx 0.1$ & Micro channel Reynolds number \\
\hline$\gamma=\rho_{p} / \rho$ & 1.25 & Density ratio \\
\hline$\theta=N^{2} D^{2} /(g(\gamma-1) d)$ & 9.08 & Shields number \\
\hline$\sigma=\frac{E I_{c s}}{\rho(\gamma-1) g d^{5}}$ & $0.5-50$ (settling) & Gravity-based flexibility number \\
\hline$\chi \phi\rangle=\frac{n V_{p}}{H T^{2}}$ & $5-150$ (mixing) & \\
\hline$\chi \nu U \ell^{2} d$ \\
$E I_{c s}$ & 0.15 & Overall solids volume fraction in mixing tank \\
\hline
\end{tabular}


Table 2: Collisional force equations and parameter settings [6]; all parameters in lattice units: unit of length is the grid spacing $\Delta$, unit if time is the time step $\Delta t$, unit of mass is the average mass per $\Delta^{3}$ (cubic) lattice cell.

\begin{tabular}{|c|c|}
\hline normal elastic force ${ }^{\mathrm{a}, \mathrm{d}}$ & $\mathbf{F}_{\mathbf{i j}}^{\mathbf{e l}}=k\left(\delta_{0}-\delta\right) \frac{\left|\delta_{\lambda}-\lambda\right|}{\lambda} \frac{\left(\mathbf{n}_{\mathbf{j}}-\mathbf{n}_{\mathbf{i}}\right)}{\left|\mathbf{n}_{\mathbf{j}}-\mathbf{n}_{\mathbf{i}}\right|}$ if $\delta<\delta_{0}$ and $\left|\delta_{\lambda}\right|<\lambda ; \quad \mathbf{F}_{\mathbf{i j}}^{\mathbf{e l}}=\mathbf{0}$ otherwise \\
\hline & $k=5.0 ; \delta_{0}=0.50 ; \lambda=0.50$ \\
\hline normal lubrication force ${ }^{b, d}$ & $\mathbf{F}_{\mathbf{i j}}^{\mathbf{n}}=k^{n}\left(\frac{1}{\delta^{*}}-\frac{1}{\delta_{d}}\right) \frac{\left|\delta_{\lambda}-\lambda\right|}{\lambda} \Delta \mathbf{u}^{\mathbf{n}}$ if $\delta^{*}<\delta_{d}$ and $\left|\delta_{\lambda}\right|<\lambda ; \quad \mathbf{F}_{\mathbf{i j}}^{\mathbf{n}}=\mathbf{0}$ otherwise \\
\hline & $k^{n}=5.0 ; \delta_{d}=1.0 ; \delta_{s a t}=0.20$ \\
\hline tangential lubrication force ${ }^{\mathrm{c}, \mathrm{d}}$ & $\mathbf{F}_{\mathbf{i j}}^{\mathbf{t}}=k^{t}\left(\frac{1}{\delta^{*}}-\frac{1}{\delta_{d}}\right) \frac{\left|\delta_{\lambda}-\lambda\right|}{\lambda} \Delta \mathbf{u}^{\mathbf{t}}$ if $\delta^{*}<\delta_{d}$ and $\left|\delta_{\lambda}\right|<\lambda ; \quad \mathbf{F}_{\mathbf{i j}}^{\mathbf{t}}=\mathbf{0}$ otherwise \\
\hline & $k^{t}=0.50$ \\
\hline $\begin{array}{l}\text { a the force on marker point } i \mathrm{w} \\
\text { normal distance } \delta \text { and tange }\end{array}$ & $\begin{array}{l}\text { h normal } \mathbf{n}_{\mathbf{i}} \text { due to marker point } j \text { on a different particle with normal } \mathbf{n}_{\mathbf{j}} \text { at } \\
\text { tial distance } \delta_{\lambda}\end{array}$ \\
\hline $\begin{array}{l}{ }^{\mathrm{b}} \text { the force on point } i \text { due to po } \\
\text { between the two points } \Delta \mathbf{u}^{\mathrm{n}} \text {; }\end{array}$ & $\begin{array}{l}j \text { on a different particle due to the relative velocity in normal direction } \\
\delta^{*}=\delta \text { if } \delta>\delta_{\text {sat }} \text { and } \delta^{*}=\delta_{\text {sat }} \text { if } \delta \leq \delta_{\text {sat }}\end{array}$ \\
\hline $\begin{array}{l}{ }^{\mathrm{c}} \text { the force on point } i \text { due to po } \\
\text { between the two points } \Delta \mathbf{u}^{\mathbf{t}}\end{array}$ & $\mathrm{t} j$ on a different particle due to the relative velocity in tangential direction \\
\hline $\begin{array}{l}\mathrm{d} \text { definition of the normal and } 1 \\
\text { ranrinted frnm } 151 \\
\left(\mathbf{n}_{2}-\mathbf{n}_{1}\right)\end{array}$ & eral distances $\delta$ and $\delta_{\lambda}$ between two adjacent marker points; figure \\
\hline
\end{tabular}

